\title{
Distance Rationalization of Voting Rules
}

\author{
Edith Elkind · Piotr Faliszewski • Arkadii \\ Slinko
}

the date of receipt and acceptance should be inserted later

\begin{abstract}
The concept of distance rationalizability allows one to define new voting rules or "rationalize" existing ones via a consensus class of elections and a distance. A candidate is declared an election winner if she is the consensus candidate in one of the nearest consensus elections. It is known that many classic voting rules are defined or can be represented in this way. In this paper, we focus on the power and the limitations of the distance rationalizability approach. We first show that if we do not place any restrictions on the class of consensus profiles or possible distances then essentially all voting rules become distance-rationalizable. Thus, to make the concept of distance ratioanalizability meaningful, we have to restrict the class of distances involved. To this end, we present a very natural class of distances, which we call votewise distances. We investigate which voting rules can be rationalized via votewise distances and study the properties of such rules and study their relations with rules that are maximumlikelyhood estimators.
\end{abstract}

\section{Introduction}

Preference aggregation is an important task both for human societies and for multiagent systems. Indeed, it is often the case that a group of agents has to make a joint decision, e.g., to select a unique alternative from a set of options available to them, even though the agents may have different opinions about the relative merits of these alternatives. A standard method of preference aggregation is voting. The agents submit ballots, which are usually rankings (total orders) of the alternatives, and a voting rule is used to select the "best" alternative. While in such settings the goal is usually to select

Edith Elkind

Division of Mathematical Sciences, School of Physical and Mathematical Sciences, Nanyang Technological University, Singapore.

Piotr Faliszewski

Department of Computer Science, AGH University of Science and Technology, Kraków, Poland. Tel.: +48-12-6173689, Fax.: +48-12-6339406, E-mail: faliszew@agh.edu.pl

Arkadii Slinko

Department of Mathematics, University of Auckland, Auckland, New Zealand. 
the alternative that reflects the individual preferences of voters in the best possible way, there is no universal agreement on how to achieve this. Any voting rule represents an agreement in the society about what should be considered as a fair outcome. This may differ from one society to another. As a consequence, there is a multitude of voting rules, and these rules are remarkably diverse (see, e.g., Brams and Fishburn (2002)).

Another reason for this diversity is the strikingly long list of impossibility theoremsstarting with the famous Arrow's impossibility theorem Arrow (1951 (revised editon, 1963)) - which state that there is no voting rule (or a social welfare function) that simultaneously satisfies several natural desiderata. Thus in each real-life scenario an election designer have to decide which of the desired conditions should be sacrificed.

An earlier view, initiated by Marquis de Condorcet, is that a voting rule must be a method for aggregating information. There is an objectively best choice but voters have different opinions because they make errors of judgment; absent these errors, they would all agree on the best choice. The goal is to design a voting rule that identifies the best choice with highest probability. This approach is called maximum likelihood estimation and it has been actively pursued in Young (1977), and in Young and Levenglick (1978) who showed that consistent application of Condorcet's ideas leads to the Kemeny rule (Kemeny, 1959). It has been shown since then that several other voting rules can be obtained as maximum likelihood estimators for different models of errors (see Conitzer, Rognlie, and Xia (2009) and Conitzer and Sandholm (2005)).

The third approach that has emerged recently in a number of papers by Nitzan (1981), Baigent (1987) and Meskanen and Nurmi (2008); can be called consensus-based. The result of each election is viewed as an imperfect approximation to some kind of electoral consensus. The winner of a given election, or a preference profile, is there fore most likely to be the undisputed winner in the "closest" consensus preference profile. The differences among voting rules can then be explained by the fact that there are several ways of defining consensus, as well as several ways of defining closeness. The heart of this approach is the agreement which situations should be viewed as "electoral consensuses," be it the existence of Condorcet winner, unanimous agreement on which candidate is best, or something else. The concept of closeness should also be agreed upon.

In this paper we concentrate on the third approach. To date, the most complete list of distance-rationalizable rules is provided by Meskanen and Nurmi (2008) (but see also Baigent (1987); Klamler (2005b, 2005a)). There, the authors show how to distance-rationalize many voting rules, including, among others, Plurality, Borda, Veto, Copeland, Dodgson, Kemeny, Slater, and STV. In the aforementioned paper Meskanen and Nurmi attempted to give a distance-rationalization for Young's rule. However it appeared to be incorrect: the authors of this paper in (Elkind, Faliszewski, \& Slinko, 2009, 2011) constructed a counterexample and gave a correct distance-rationalization for Young's rule, which appeared to be highly nontrivial.

In Section 3.3 we show that every reasonable voting rule can be distance rationalized with respect to some distance and some notion of consensus. This indicates that the notion of distance rationalizability used in the extant work is too broad to be meaningful. Hence, we have to determine what are the "reasonable" consensus classes and the "reasonable" distances and to reexamine all existing results from this perspective.

In Section 4 we suggest a family of "good" distances (which we call votewise distances) and study voting rules that are distance rationalizable with respect to such distances. In particular, in Section 4.2 we show that many of the rules considered in 
Meskanen \& Nurmi (2008), as well as all scoring rules and a variant of the Bucklin rule, can be rationalized via distances from this family. In contrast, we demonstrate that STV, which was shown to be distance-rationalizable in (Meskanen \& Nurmi, 2008), is not distance-rationalizable via votewise distances, i.e., the restricted notion of distance rationalizability has indeed some bite.

Distance rationalizability can be used as an alternative to an axiomatic characterization of voting rules. In this case we impose axioms not on the rule as a whole but on underlying distances and consensus classes. In particular, in Section 4.5 we obtain such a characterization of scoring voting rules which we hope will be as useful as the famous Young's axiomatic characterization (Young, 1975) has been.

Importantly, the distance rationalizability framework can be viewed as a general method for specifying and analyzing voting rules. As such, it may be useful for proving results for entire families of voting rules, rather than isolated rules. For instance, a lot of recent research in computational social choice has focused on the complexity of determining (possible) election winners (see, e.g., E. Hemaspaandra, Hemaspaandra, and Rothe (1997); Konczak and Lang (2005)), and the complexity of various types of attacks on elections (e.g., manipulation Conitzer, Sandholm, and Lang (2007), bribery Faliszewski, Hemaspaandra, and Hemaspaandra (2009), and control Meir, Procaccia, Rosenschein, and Zohar (2008); Faliszewski, Hemaspaandra, Hemaspaandra, and Rothe (2009)). ${ }^{1}$ However, most of the results in this line of work are specific to particular voting rules. We believe that the ability to describe multiple voting rules in a unified way (e.g., via the distance rationalizability framework) will lead to more general results. To provide an argument in favor of this belief, in Sections 4.1 and Section 5 we present initial results of this type, relating the type of distance and consensus used to rationalize a voting rule with the complexity of winner determination under this rule as well as the rule's axiomatic properties (such as anonymity, neutrality and consistency).

Finally we compare the distance rationalizability and maximum likelihood estimation frameworks and conclude that the former is more general and more flexible. All the rules that are maximum likelihood estimators for well-behaved noise models can be also obtained through distance rationalizability. Our results imply that Litvak's rule is a new maximum likelihood estimator rule.

\section{Preliminaries: elections, distances and consensus classes}

\subsection{Elections}

An election is a pair $E=(C, V)$ where $C$ is the set of candidates and $V=$ is an ordered list of voters. Each voter is represented by her vote, i.e., a strict, linear order over the set of candidates (also called a preference order). We will refer to the list $V$ as a preference profile, and we denote the number of voters in $V$ by $|V|$. The number of alternatives will be denoted by $|C|$.

A voting rule $\mathcal{R}$ is a function that given an election $E=(C, V)$ returns a non-empty set of election winners $\mathcal{R}(E) \subseteq C$. Note that it is legal for the set of winners to contain more that one candidate. When the set of candidates is clear, we will sometimes write

\footnotetext{
1 These references are only examples; an overview of literature is far beyond the scope of this paper.
} 
$\mathcal{R}(V)$ instead of $\mathcal{R}(E)$. We sometimes consider voting rules defined for a particular number of candidates (or even a particular set of candidates) only.

Below we define several prominent voting rules that would be mentioned later.

Scoring rules. For any $m$-element vector $\alpha=\left(\alpha_{1}, \ldots, \alpha_{m}\right)$ of nonnegative integers, where $\alpha_{1} \geq \alpha_{2} \geq \ldots \geq \alpha_{m}$, a scoring rule $\mathcal{R}_{\alpha}$ is defined as follows. Fix an election $E=(C, V)$, where $C=\left\{c_{1}, \ldots, c_{m}\right\}$ and $V=\left(v_{1}, \ldots, v_{n}\right)$. The $\alpha$-score of

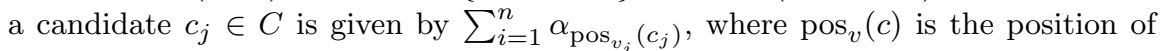
candidate $c$ in $v$ 's ranking. That is, candidate $c_{j}$ receives $\alpha_{i}$ points from each voter that puts her in the $i$ th position. The winners of $E$ under $\mathcal{R}_{\alpha}$ are the candidates with the maximum $\alpha$-score. Note that a scoring rule is defined for a fixed number of candidates. However, many standard voting rules can be defined via families of scoring rules. For example, Plurality is defined via the family of vectors $(1,0, \ldots, 0)$, veto is defined via the family of vectors $(1, \ldots, 1,0)$, and Borda is defined via the family of vectors $(m-1, m-2, \ldots, 0) ; k$-approval is the scoring rule with $\alpha_{i}=1$ for $i \leq k, \alpha_{i}=0$ for $i>k$.

Bucklin and Simplified Bucklin. Given a positive integer $k, 1 \leq k \leq|C|$, we say that a candidate $c$ is a $k$-majority winner if more than $\frac{|V|}{2}$ voters rank $c$ among the top $k$ candidates. Let $k^{\prime}$ be the smallest positive integer such that there is at least one $k^{\prime}$-majority winner for $E$. The Bucklin score of a candidate $c$ is the number of voters that rank her in top $k^{\prime}$ positions. The Bucklin winners are the candidates with the highest Bucklin score; clearly, all of them are $k^{\prime}$-majority winners. The simplified Bucklin winners are all $k^{\prime}$-majority winners.

Single Transferable Vote (STV). In STV the winner is chosen by repeated elimination of "inferior" candidates. We find a candidate with the lowest Plurality score (i.e., one that is ranked first the least number of times) and remove her from the votes (this may require tie-breaking). We repeat the process until a single candidate remains; this candidate is declared to be the winner. For STV the issue of handling ties - that is, the issue of the order in which candidates with lowest Plurality scores are deleted - is quite important, and is discussed in detail by Conitzer, Rognlie and Xia (Conitzer et al., 2009). However, the results in our paper are independent of the tie-breaking rule.

Dodgson. A Condorcet winner is a candidate that is preferred to any other candidate by a majority of voters. The score of a candidate $c$ is the smallest number of swaps of adjacent candidates that have to be performed on the votes to make $c$ a Condorcet winner. The winner(s) are the candidate(s) with the lowest score.

Kemeny. Let $u$ and $v$ be two preference orders over $C$. The number of disagreements between $u$ and $v$, denoted $t(u, v)$, is the number of pairs of candidates $c_{i}, c_{j}$ such that either $c_{i}$ is ranked higher than $c_{j}$ in $u$ and $c_{j}$ is ranked higher than $c_{i}$ in $v$ or the vice versa. A candidate is a Kemeny winner of the profile $\left(v_{1}, \ldots, v_{n}\right)$ if it is ranked first in any preference order $v$ that minimizes the $\operatorname{sum} \sum_{i=1}^{n} t\left(v, v_{i}\right)$.

Litvak. Litvak's method (Litvak, 1982; Meskanen \& Nurmi, 2008) is similar to Kemeny rule. If $|C|=m$, then each preference ranking $v$ is assigned a vector $\ell(v)$ of $m$ components. The component $i$ of this vector indicates how many alternatives are placed in $v$ ahead of $c_{i}$. In other words

$$
\ell(v)=\left(\operatorname{pos}_{v}\left(c_{1}\right), \operatorname{pos}_{v}\left(c_{2}\right), \ldots, \operatorname{pos}_{v}\left(c_{m}\right)\right) .
$$

Each such vector is thus a permutation of numbers $0, \ldots, m-1$. Given two such vectors $p=\left(p_{1}, \ldots, p_{m}\right)$ and $q=\left(q_{1}, \ldots, q_{m}\right)$, we define the disagreement between 
them as $s(p, q)=\sum_{i=1}^{n}\left|p_{i}-q_{i}\right|$. Given a profile $\left(v_{1}, \ldots, v_{n}\right)$, a candidate is a Litvak winner of the election $E=(V, C)$ if it is ranked first in any preference order $v$ that minimizes the sum $\sum_{i=1}^{n} s\left(\ell(v), \ell\left(v_{i}\right)\right) .^{2}$

Threshold. Given a profile $V=\left(v_{1}, \ldots, v_{n}\right)$ and a candidate $c$ the threshold rule calculates score of $c$ as the the lowest rank of $c$ in orders of $V$ i.e., the score of $c$ is $\max _{i=1}^{n}\left(\operatorname{pos}_{v_{i}}(c)\right)$. Then the set of threshold winners of the profile $V$ are the set of alternatives with the lowest score. Instead we can calculate the score of $c$ as $\min _{i=1}^{n}\left(m-\operatorname{pos}_{v_{i}}(c)\right)$ and then select winners as the alternatives with the maximal score. The latter formulation reminds us that the threshold rule is a distant relative of the Borda rule. Indeed, instead of summing the values $m-\operatorname{pos}_{v_{i}}(c)$ up to obtain the Borda score we take a minimum. This rule was introduced by Aleskerov, Chistyakov, and Kalyagin (2010) (see also references there).

\subsection{Distances}

Let $X$ be a set. A function $d: X \times X \rightarrow \mathbb{R} \cup\{\infty\}$ is a distance (or, a metric) if for each $x, y, z \in X$ it satisfies the following four conditions:

(a) $d(x, y) \geq 0$ (nonnegativity),

(b) $d(x, y)=0$ if and only if $x=y$ (identity of indiscernibles),

(c) $d(x, y)=d(y, x)$ (symmetry),

(d) $d(x, y) \leq d(x, z)+d(z, y)$ (triangle inequality).

If $d$ satisfies all of the above conditions except the second one (identity of indiscernibles) then $d$ is called a pseudodistance.

We are going to use distances on profiles to define voting rules. For all voting procedures acceptable for a democratic society the outcomes must depend on the votes cast in a simple and transparent manner. In mathematical language this means that any distance between profiles must be a function of linear orders from those profiles. Therefore a natural approach would be to define a distance on linear orders first and then try to extend it to a distance over the profiles.

Let $P(C)$ be the set of all possible linear orders (votes) over a set of candidates $C$. Two particularly useful distances over votes are the discrete distance and the swap distance defined below. Let $u$ and $v$ be two linear orders over $C$. Here by $u(i)$ we will denote the alternative ranked $i$ th in $u$.

Discrete distance. The discrete distance $d_{\text {discr }}(u, v)$ is defined to be 0 if $u=v$ and 1 otherwise.

Swap distance. The swap distance ${ }^{3} d_{\text {swap }}(u, v)$ is the least number of swaps of adjacent candidates that transform linear order $u$ into linear order $v$.

Sertel distance. The Sertel's distance is defined as

$$
d_{\text {sert }}(u, v)=\max _{i}[u(i) \neq v(i)]
$$

2 Bury and Wagner (2003) give a concise discussion on Litvak's method and provide computational algorithms for determining of what they call the Litvak median.

3 also called Kendall tau distance, Dodgson distance and bubble-sort distance. 
This distance is non-Archimedean (ultrametric) and satisfies the ultrametric inequality

$$
d_{\text {sert }}(u, w) \leq \max \left[d_{\text {sert }}(u, v), d_{\text {sert }}(v, w)\right] .
$$

Spearman's distance and generalizations. The Spearman's distance ${ }^{4}$ between the linear orders $u$ and $v$ is

$$
d_{\text {spear }}(u, v)=\sum_{i=1}^{m}\left|\operatorname{pos}_{u}\left(c_{i}\right)-\operatorname{pos}_{v}\left(c_{i}\right)\right| .
$$

An interesting modification of this distance appears when we take in (2) maximum instead of the sum:

$$
d_{\text {spear }}^{\infty}(u, v)=\max _{i=1}^{m}\left|\operatorname{pos}_{u}\left(c_{i}\right)-\operatorname{pos}_{v}\left(c_{i}\right)\right| .
$$

We may generalize Spearman's distance by assuming that dscrepancies in different positions have different costs. Let $\alpha=\left(\alpha_{1}, \ldots, \alpha_{m}\right)$ be any vector with nonnegative coordinates. We define a new distance between two orders $u$ and $v$ as

$$
d_{\alpha}(u, v)=\sum_{i=1}^{m}\left|\alpha_{\operatorname{pos}_{u}\left(c_{i}\right)}-\alpha_{\operatorname{pos}_{v}\left(c_{i}\right)}\right| .
$$

Note that if we obtain $v$ from $u$ by swapping elements in positions $i$ and $j$, the distance of the new order from the old one will be $2\left|\alpha_{i}-\alpha_{j}\right|$. In general, some several weights may be equal and in this case $d_{\alpha}$ is a pseudodistance.

There are a number of other natural distances on $P(C)$ used in statistics, computer science and biology, some of them are computationally involved. For example, calculating the reversal distance defined to be the number of substring reversals required to transform one linear order into another is NP-hard (Carpara, 1977) There is an Encyclopedia of Distances (Deza \& Deza, 2009).

Now we have to extend a given distance on $P(C)$ to a distance on the set of profiles $P(C)^{n}$, where $n=|V|$. This can be done in many different ways and we are very much in a situation similar to the functional analysis, when it concerns with introducing norms in spaces of dimension larger than one.

Any distance over votes can be extended in several ways to the distance over the profiles. Let $d$ be a distance on $P(C)$ and let $E=(C, U)$ and $E^{\prime}=(C, V)$ be two elections with $U=\left(u_{1}, \ldots, u_{n}\right)$ and $V=\left(v_{1}, \ldots, v_{n}\right)$ being two profiles from $P(C)^{n}$. Then we define the $l^{k}$ metric on elections by

$$
\widehat{d}^{k}\left(E, E^{\prime}\right)=\left(\sum_{i=1}^{n} d\left(u_{i}, v_{i}\right)^{k}\right)^{\frac{1}{k}}, \quad \widehat{d}^{\infty}\left(E, E^{\prime}\right)=\max _{i} d\left(u_{i}, v_{i}\right)
$$

(and we set $\widehat{d}\left(E^{\prime}, E^{\prime \prime}\right)=\infty$ if the candidate sets are different or the profiles have different number of voters).

Clearly, $\widehat{d}^{k}, k=1,2, \ldots, \infty$, satisfies all distance axioms as long as $d$ does. $l^{1}$ and $l^{2}$ metrics have been widely used in preference aggregation literature, see, for example, (Bogard, 1973, 1975; Cook \& Seiford, 1978, 1982; Litvak, 1983) although $l^{2}$ has not been used yet in context of distance rationalizability. However, further we will need

4 known in statistics as "Spearman's footrule" (Kendall \& Gibbons, 1990), also called Litvak's distance 
$l^{\infty}$ metric to distance rationalize Bucklin and Threshold rules. Since $\widehat{d}^{1}$ has by far the most frequent appearance we will omit 1 in this case and write $\widehat{d}$ if this may not lead to a confusion.

\subsection{Consensus classes}

Intuitively, we say that an election $E=(C, V)$ is a consensus if it has an undisputed winner reflecting a certain concept of fairness in the society. Formally, a consensus class is a pair $(\mathcal{E}, \mathcal{W})$ where $\mathcal{E}$ is a set of elections and $\mathcal{W}: \mathcal{E} \rightarrow C$ is a mapping which for each election $E \in \mathcal{E}$ assigns a unique alternative, which is called the consensus alternative (winner). The following four natural classes have been historically used by societies as situations of consensus:

Strong unanimity. Denoted $\mathcal{S}$, this class contains elections $E=(C, V)$ where all voters report the same preference order. The consensus alternative $\mathcal{W}(E)$ is the candidate ranked first by all the voters.

Unanimity. Denoted $\mathcal{U}$, this class contains all elections $E=(C, V)$ where all voters rank some candidate $c$ first. The consensus alternative $\mathcal{W}(E)$ of such an election is $c$.

Majority. Denoted $\mathcal{M}$, this class contains all elections $E=(C, V)$ where more than half of the voters rank some candidate $c$ first. The consensus alternative $\mathcal{W}(E)$ of such an election is $c$.

Condorcet. Denoted $\mathcal{C}$, this class contains all elections $E=(C, V)$ with a Condorcet winner (defined above). The Condorcet winner is the consensus alternative $\mathcal{W}(E)$.

There are other quite natural classes that could but yet have not been used to define a consensus in the society. One of them is based on the concept of Lorenz dominance of vectors. Given two vectors $x=\left(x_{1}, \ldots, x_{n}\right)$ and $y=\left(y_{1}, \ldots, y_{n}\right)$ we say that $x$ Lorenz dominates $y$ if $\sum_{i=1}^{k} x_{i} \geq \sum_{i=1}^{k} y_{i}$ for all $k=1,2, \ldots, n$ and the inequality is strict for at least one $k$. Given a profile $V=\left(v_{1}, \ldots, v_{n}\right)$ over $C$, for each alternative $c \in C$ we define a vector $\ell(c)$ whose $i$ th coordinate shows how many times $c$ was ranked $i$ th or higher in linear orders of $V$. Am alternative $c$ will be called a Lorenz winner ${ }^{5}$ if $\ell(c)$ Lorenz dominates $\ell(d)$ for all $d \in C$ different from $c$. So we define the following consensus class:

Lorenz. Denoted $\mathcal{L}$, this class contains all elections $E=(C, V)$ with a Lorenz winner. The Lorenz winner is the consensus alternative $\mathcal{W}(E)$.

This natural consensus class is virtually unused and unstudied. It is different from all four classes introduced earlier. To demonstrate this we give the following example. Consider the following profile of 15 voters on the set of three alternatives $a, b, c$

\begin{tabular}{lllll}
3 & 3 & 2 & 5 & 2 \\
\hline$a$ & $a$ & $b$ & $b$ & $c$ \\
$b$ & $c$ & $a$ & $c$ & $a$ \\
$c$ & $b$ & $c$ & $a$ & $b$
\end{tabular}

5 We thank Jérôme Lang for attracting our attention to this concept. 
the Condorcet winner is $a$ while the Lorenz winner is $b$. Indeed, $\ell(a)=(6,10,15)$ and $\ell(b)=(7,10,15)$.

Also, one can certainly consider situations in which the voters reach a consensus that several candidates are equally well qualified to be elected (this may happen, for example, in Approval voting when all voters approve two or more candidates). However, in this paper we limit ourselves to consensuses with unique winners.

\section{Distance rationalizability}

\subsection{An Attempt at a Definition and the Results So Far}

Firstly, we show how the consensus class and a distance define a voting rule.

Definition 1 Let $d$ be a distance over elections and let $\mathcal{K}=(\mathcal{E}, \mathcal{W})$ be a consensus class. We define the $(\mathcal{K}, d)$-score of a candidate $c$ in an election $E$ to be the distance (according to $d$ ) between $E$ and a closest consensus election $E^{\prime} \in \mathcal{E}$ such that $c$ is a winner of $E^{\prime}$. The set of $(\mathcal{K}, d)$-winners of an election $E=(C, V)$ consists of those candidates in $C$ whose $(\mathcal{K}, d)$-score is the smallest.

We can now formally define the concept of distance rationalizability of a voting rule.

Definition 2 A voting rule $\mathcal{R}$ is distance-rationalizable via a consensus class $\mathcal{K}$ and a distance $d$ over elections, if for each election $E$, a candidate $c$ is an $\mathcal{R}$-winner of $E$ if and only if she is a $(\mathcal{K}, d)$-winner of $E$. We also say that $\mathcal{R}$ is $(\mathcal{K}, d)$-rationalizable.

Many common voting rules are known to be distance-rationalizable in a very natural way. Nitzan $(1981)$ proved that Plurality is $\left(\mathcal{U}, \widehat{d}_{\text {discr }}\right)$-rationalizable and Borda rule is $\left(\mathcal{U}, \widehat{d}_{\text {swap }}\right)$-rationalizable. For many rules their distance rationalizations are obvious from their definitions, e.g., Kemeny rule is $\left(\mathcal{S}, \widehat{d}_{\text {swap }}\right)$-rationalizable, and Dodgson rule is $\left(\mathcal{C}, \widehat{d}_{\text {swap }}\right)$-rationalizable. It is quite remarkable that these three major voting rules - Borda, Dodgson and Kemeny - can be rationalized using the same distance. Meskanen and Nurmi (2008) show that Litvak's rule is $\left(\mathcal{S}, \widehat{d}_{\text {spear }}\right)$-rationalizable. They also distance-rationalized STV but the distance used by them is extremely complicated; in Section 4 we will discover the reason for this complexity.

Meskanen and Nurmi (2008) claim that they have distance rationalized Young's rule. However, as the authors of this paper shown (Elkind et al., 2009, 2011), they actually distance-rationalized another rule, which had previously no name and dubbed it voters-replacement rule. Elkind et al. $(2009,2011)$ give a correct distance rationalization of Young's rule and distance-rationalize Minimax.

Also Meskanen and Nurmi (2008) consider a number of rules whose definition use outranking matrices or the majority relation, e.g., Slater rule or Schulze's (2003) method. However distances they obtain in this case are actually pseudodistances as different profiles (and even voting situations) can lead to the same outranking matrix.

We remark that the notion of distance-rationalizability introduced in Definition 2 allows for arbitrary consensus classes and distances; as we will see in the next section, this lack of constraints results in a definition that is too broad to be useful as any rule can be distance-rationalized. Distance rationalizability approach turns thus into a 
program of classification of voting rules by two parameters: a distance and a consensus class and the key question is: Which rules can be rationalized by using natural distances and natural consensus classes? A good distance rationalization of a rule provides an undisputed value. In the next section we further contribute to this program by analyzing arbitrary scoring rules from this perspective.

\subsection{Scoring Rules}

In this section, we will show that all scoring rules - an important class of voting rules that includes such famous rules as Plurality, Borda, Veto, and $k$-approval-are very close to being distance-rationalizable. More precisely, we prove that all such rules are pseudodistance-rationalizable, i.e., can be defined via a consensus class and a pseudodistance.

Any vector $\alpha=\left(\alpha_{1}, \ldots, \alpha_{m}\right)$ can be used to define a pseudodistance $d_{\alpha}$ over profiles of preferences over candidates in $C$ with $|C|=m$ as follows. For any two voters $v$ and $w$ set

$$
d_{\alpha}(v, w)=\sum_{i=1}^{m}\left|\alpha_{\operatorname{pos}_{v}\left(c_{i}\right)}-\alpha_{\operatorname{pos}_{w}\left(c_{i}\right)}\right| .
$$

It is not hard to see that $d_{\alpha}$ satisfies all pseudodistance axioms. Moreover, if $\alpha_{i} \neq \alpha_{i+1}$ for all $i=1, \ldots, m-1$ (we will call such vectors, and the corresponding distances and scoring rules faithful), then $d_{\alpha}$ also satisfies axiom (2), i.e., it is a distance. On the other hand, if $\alpha_{i}=\alpha_{i+1}$ for some $i=1, \ldots, m$, then we have $d_{\alpha}(v, w)=0$ for the two voters $v, w$ such that $w$ is obtained from $v$ by swapping the $i$ th and the $(i+1)$ st candidate in $v$ 's preference ordering, and $d_{\alpha}$ is indeed only a pseudodistance, but not a distance. Note that for $\alpha=(m-1, \ldots, 1,0)$ - the vector that corresponds to the Borda rule - we have $d_{\alpha}(v, w)=2 d_{\text {swap }}(v, w)$. More generally, $d_{\alpha}(v, w)$ can be interpreted as the cost of transforming $v$ into $w$ by a sequence of swaps of adjacent candidates, where the cost of swapping the candidate in the $k$ th position with the one just below him is given by $2\left(\alpha_{k}-\alpha_{k+1}\right)$.

We will now prove that any scoring rule $\mathcal{R}_{\alpha}$ is $\left(\mathcal{U}, \widehat{d_{\alpha}}\right)$-rationalizable, where $\widehat{d_{\alpha}}$ is the pseudodistance over elections that corresponds to $d_{\alpha}$. Note that this implies that all faithful scoring rules are distance rationalizable. While distance rationalizability of the Borda rule was proven by Nitzan (1981), no such result was previously known for other scoring rules.

Theorem 1 For each scoring vector $\alpha=\left(\alpha_{1}, \ldots, \alpha_{m}\right)$ and each election $E=(C, V)$ with $|C|=m$, a candidate $c \in C$ is a winner of $E$ according to $\mathcal{R}_{\alpha}$ if and only if $c$ is $a\left(\mathcal{U}, \widehat{d_{\alpha}}\right)$-winner of $E$.

Proof Fix a candidate $c_{j} \in C$, and consider a voter $v \in V$ that ranks $c_{j}$ in the $k$ th position, i.e., $\operatorname{pos}_{v}\left(c_{j}\right)=k$. Consider an arbitrary preference order $w$ in which $c_{j}$ is ranked first. We have $\sum_{\ell=1}^{m} \alpha_{\operatorname{pos}_{v}\left(c_{\ell}\right)}=\sum_{\ell=1}^{m} \alpha_{\operatorname{pos}_{w}\left(c_{\ell}\right)}=\sum_{i=1}^{m} \alpha_{i}$, so $\sum_{\ell=1}^{m}\left(\alpha_{\operatorname{pos}_{w}\left(c_{\ell}\right)}-\right.$ $\left.\alpha_{\operatorname{pos}_{v}\left(c_{\ell}\right)}\right)=0$. On the other hand, we have $\alpha_{\operatorname{pos}_{w}\left(c_{j}\right)}-\alpha_{\operatorname{pos}_{v}\left(c_{j}\right)}=\alpha_{1}-\alpha_{k}$. For any $m$ real numbers $a_{1}, \ldots, a_{m}$ such that $\sum_{i=1}^{m} a_{i}=0$ we have $\sum_{i=1}^{m}\left|a_{i}\right| \geq\left|a_{j}\right|+\left|\sum_{i \neq j} a_{i}\right|=$ $2\left|a_{j}\right|$. Thus, for $d_{\alpha}(v, w)$ given by (5) we have

$$
d_{\alpha}(v, w) \geq 2\left|\alpha_{\operatorname{pos}_{w}\left(c_{j}\right)}-\alpha_{\operatorname{pos}_{v}\left(c_{j}\right)}\right|=2\left(\alpha_{1}-\alpha_{k}\right) .
$$


On the other hand, for the preference order $w^{\prime}$ that is obtained from $v$ by swapping $c_{j}$ with the top candidate in $v$, we have $d_{\alpha}\left(v, w^{\prime}\right)=2\left(\alpha_{1}-\alpha_{k}\right)$. Hence, the $d_{\alpha}$-distance from $v$ to the nearest vote that ranks $c_{j}$ first is exactly $2\left(\alpha_{1}-\alpha_{k}\right)$, and, consequently, the $\widehat{d_{\alpha}}$-distance from $E$ to the nearest unanimous election in which all voters rank $c_{j}$ first is exactly $\sum_{i=1}^{n} 2\left(\alpha_{1}-\alpha_{\operatorname{pos}_{v_{i}}\left(c_{j}\right)}\right)$. On the other hand, the score that $c_{j}$ receives

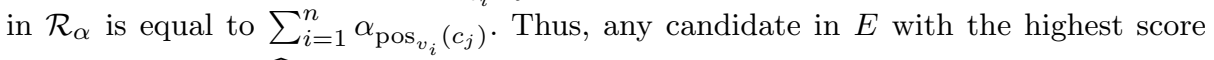
under $\mathcal{R}_{\alpha}$ is a $\left(\mathcal{U}, \widehat{d_{\alpha}}\right)$-winner of $E$ and vice versa.

For some scoring rules the statement of Theorem 1 cannot be strengthened to distance rationalizability.

Proposition 1 Any scoring rule defined by a vector $\alpha$ with $\alpha_{1}=\alpha_{2}$ is not distancerationalizable with respect to consensus class $\mathcal{U}$.

Proof Fix a scoring rule $\mathcal{R}_{\alpha}$ with $\alpha_{1}=\alpha_{2}$. Consider an election $E$ in which all voters rank some candidate $c_{1}$ first and another candidate $c_{2}$ second. Under $\mathcal{R}_{\alpha}$, both $c_{1}$ and $c_{2}$ are winners. On the other hand, $E$ is clearly unanimous, with $c_{1}$ being the consensus winner. Thus, for any distance $d$, the distance between $E$ and the closest election in $\mathcal{U}$ is 0 . Therefore, for $c_{2}$ to be a $(\mathcal{U}, d)$-winner, there must exist a unanimous election $E^{\prime}$ in which $c_{2}$ is ranked first such that $d\left(E, E^{\prime}\right)=0$. However, as $E^{\prime}$ is necessarily different from $E$, this is impossible for any distance $d$.

Observe that the condition of Proposition 1 is satisfied by Veto and $k$-approval for $k>1$, so these rules are not distance-rationalizable with respect to $\mathcal{U}$.

What about scoring rules with $\alpha_{1} \neq \alpha_{2}$, but $\alpha_{j}=\alpha_{j+1}$ for some $j=2, \ldots, m-1$ ? As argued above, for such rules $d_{\alpha}$ is a pseudodistance, but not a distance. It is tempting to conjecture that we can extend the proof of Proposition 1 to this case in order to show that no such rule is distance-rationalizable with respect to $\mathcal{U}$. However, this conjecture is easy to refute: indeed, the Plurality rule for $m$ candidates is a scoring rule with $\alpha_{2}=\ldots=\alpha_{m}=0$, but we have seen that Plurality can be distance-rationalized with respect to $\mathcal{U}$. Observe that the distance $d_{\text {discr }}$ that we have used for this purpose is different from $d_{(1,0, \ldots, 0)}$. However, it is not clear how to generalize this construction to other scoring rules with $\alpha_{1}>\ldots>\alpha_{j}=\alpha_{j+1}$. Thus, distance rationalizability of such rules with respect to $\mathcal{U}$ remains an open question.

We have shown that any faithful scoring rule can be rationalized with respect to the consensus class $\mathcal{U}$. We will now argue that there is no scoring rule that can be rationalized with respect to Condorcet consensus; the proof is similar to that of Proposition 1.

Proposition 2 No scoring rule is distance-rationalizable with respect to the consensus class $\mathcal{C}$.

Proof It is known that no scoring rule is Condorcet-consistent (Moulin, 1991). That is, for any scoring rule $\mathcal{R}$ there exists an election $E=(C, V)$ such that $\mathcal{R}(E)=\{c\}, E$ has a Condorcet winner $c^{\prime}$, but $c \neq c^{\prime}$. Now, consider any distance $d$. Since $d$ satisfies axiom (2) (identity of indiscernibles), the election $E$ has exactly one candidate with the $(\mathcal{C}, d)$-score of 0 , this is $c^{\prime}$. Hence, the set of $(\mathcal{C}, d)$-winners of $E$ consists of $c^{\prime}$ only, and is therefore different from the set of $\mathcal{R}$-winners of $E$. 


\subsection{Unrestricted Distance-Rationalizability}

We say that a voting rule $\mathcal{R}$ over a set of candidates $C$ satisfies nonimposition if for every $c \in C$ there exists an election with the set of candidates $C$ in which $c$ is the unique winner under $\mathcal{R}$. Clearly, nonimposition is a very weak condition, even weaker than unanimity, and is satisfied by all common voting rules. Nevertheless, it turns out to be sufficient for unrestricted distance-rationalizability.

Theorem 2 For any voting rule $\mathcal{R}$ over a set of candidates $C$ that satisfies nonimposition, there is a consensus class $\mathcal{K}=(\mathcal{E}, \mathcal{W})$ and a distance $d$ such that $\mathcal{R}$ is $(\mathcal{K}, d)$-rationalizable.

Proof Since $\mathcal{R}$ satisfied nonimposition, for each $c \in C$ there exists an election $E_{c}=$ $\left(C, V_{c}\right)$ in which $c$ is the unique winner. Define an undirected graph $G=(K, F)$ as follows. The set $K$ consists of all profiles over $C$ (note that this set is infinite). The set $F$ contains an edge between profiles $U$ and $V$ if $|\mathcal{R}(U)|=1$ and $\mathcal{R}(U) \subseteq \mathcal{R}(V)$ or $|\mathcal{R}(V)|=1$ and $\mathcal{R}(V) \subseteq \mathcal{R}(U)$. For any two elections $E_{U}=(C, U)$ and $E_{V}=(C, V)$, we define $d\left(E_{U}, E_{V}\right)$ to be the shortest path distance between $U$ and $V$ in $G$. It is easy to check that $d$ is indeed a distance.

A profile will be a consensus profile if it has a unique winner, i.e., we set $\mathcal{E}=\{E \mid$ $|\mathcal{R}(E)|=1\}$, and let the unique winner $\mathcal{W}(E)$ of the $\mathcal{E}$-consensus election $E$ be $\mathcal{R}(E)$. We set $\mathcal{K}=(\mathcal{E}, \mathcal{W})$.

Now, suppose that $E \in \mathcal{E}$. Then $d(E, E)=0$ and there does not exist an election $E^{\prime} \neq E$ such that $d\left(E, E^{\prime}\right)=0$. So the $(\mathcal{K}, d)$-winner of $E$ is $\mathcal{W}(E)=\mathcal{R}(E)$. On the other hand, if $E \notin \mathcal{E}$, then $d\left(E, E^{\prime}\right) \geq 1$ for any election $E^{\prime}$, and for any $c \in \mathcal{R}(E)$ we have $d\left(E, E_{c}\right)=1$. Moreover, for any $c \notin \mathcal{R}(E)$ and any $E^{\prime} \in \mathcal{E}$ such that $\mathcal{R}\left(E^{\prime}\right)=\{c\}$ we have $d\left(E, E^{\prime}\right) \geq 2$. Thus, the set $\mathcal{R}(E)$ is exactly the set of $(\mathcal{K}, d)$-winners of $E$. Thus $\mathcal{R}$ is $(\mathcal{K}, d)$-rationalizable.

The consensus class used in the proof of Theorem 2 is rather artificial. However, the following theorem shows that a similar result holds for our four natural consensus notions too.

Definition 3 Let $\mathcal{R}$ be a voting rule and let $\mathcal{K}=(\mathcal{E}, \mathcal{W})$ be a consensus class. We say that $\mathcal{R}$ is compatible with $\mathcal{K}$, or $\mathcal{K}$-compatible, if for each election $E$ in $\mathcal{E}$ it holds that $\mathcal{R}(E)=\mathcal{W}(E)$.

The next theorem shows that compatibility with a particular consensus is equivalent to distance-rationalizability with respect to this consensus. In what follows we prove this result for the four consensus classes considered in this paper; however, it can be generalized to any consensus class that has the property that any candidate is a consensus winner in one of the profiles of this class. Note also that any voting rule that is compatible with any such consensus class also satisfies nonimposition, so the compatibility condition is more restrictive than nonimposition.

Theorem 3 For any consensus class $\mathcal{K}=(\mathcal{E}, \mathcal{W})$, a voting rule $\mathcal{R}$ is $(\mathcal{K}, d)$-rationalizable for some distance $d$ if and only if $\mathcal{R}$ is $\mathcal{K}$-compatible.

Proof Suppose $\mathcal{R}$ is $(\mathcal{K}, d)$-rationalizable and let $E \in \mathcal{E}$. Then there is only one election at distance 0 from it-which is $E$ itself,- so the $(\mathcal{K}, d)$-winner of $E$ is $\mathcal{W}(E)$ and $\mathcal{R}$ is $\mathcal{K}$-compatible. 
For the converse let us assume that $\mathcal{R}$ is $\mathcal{K}$-compatible. To construct its distance rationalization we will use a distance very similar to the one used in Theorem 2 ; the only difference is that we require $d^{\prime}\left(E, E^{\prime}\right)=+\infty$ if $E$ and $E^{\prime}$ have a different number of voters. Let $P(C)$, as above, be the set of all possible votes over $C$. For each $n \in \mathbb{Z}^{+}$, we define an undirected graph $G(C, n)=(K, F)$, where the set of vertices $K$ is $P(C)^{n}$ i.e., the set of all possible profiles of $n$ votes over $C$, and there is an edge between $U$ and $V$ if and only if $(C, U) \in \mathcal{E}$ and $\mathcal{R}(U) \subseteq \mathcal{R}(V)$ or $(C, V) \in \mathcal{E}$ and $\mathcal{R}(V) \subseteq \mathcal{R}(U)$.

We define the distance $d\left(E, E^{\prime}\right)$ between two elections $E=(C, U)$ and $E^{\prime}=(C, V)$ to be the shortest path distance between $U$ and $V$ in $G(C, n)$ if $U$ and $V$ are both in $P(C)^{n}$ for some $n>0$, and set $d\left(E, E^{\prime}\right)=+\infty$ if $E$ and $E^{\prime}$ have a different number of voters. The argument that $\mathcal{R}$ is $(\mathcal{K}, d)$-rationalizable follows along the same lines as the proof of Theorem 2 .

Since almost all interesting rules are $\mathcal{S}$-compatible, effectively, Theorem 3 shows that almost any interesting voting rule is distance-rationalizable with respect to the strong unanimity consensus. Exceptions are those rules that do not have unique winners on strongly unanimous profiles, e.g., veto and $k$-approval (for each $k>1$ ). However, both veto and $k$-approval satisfy nonimposition and thus are distance-rationalizable via Theorem 2.

This brings us to the conclusion: knowing that a rule is distance-rationalizableeven with respect to a standard notion of consensus - provides no further insight into the properties of the rule. Moreover, the dichotomy between distance-rationalizable and non-distance-rationalizable rules becomes essentially meaningless. This observation does not, however, diminish the value of distance-rationalizations of particular rules. Distance-rationalizability approach becomes a program of classification of voting rules according to the two parameters. So any attempts to rationalize known voting rules using simpler or nicer distances and consensus classes are meaningful.

The distance employed in the proof of Theorem 3 is very unnatural and bad in many respects. In particular, as the following proposition implies, it may not be polynomialtime computable.

Proposition 3 Let $\mathcal{K}=(\mathcal{E}, \mathcal{W})$ be a consensus class and $\mathcal{R}$ be a voting rule, compatible with $\mathcal{K}$. Let $d$ be the distance defined in the proof of Theorem 3. Suppose that

(a) for any election $E$ we can check in polynomial-time if $E \in \mathcal{E}$,

(b) there is a polynomial-time algorithm for computing $d$,

(c) the rule $\mathcal{W}$ on $\mathcal{E}$ satisfies nonimposition, moreover for an element $c \in C$ we can in polynomial time construct a profile $E_{c} \in \mathcal{E}$ such that $\mathcal{W}\left(E_{c}\right)=c$.

Then we can construct a polynomial-time algorithm that solves the winner determination problem for $\mathcal{R}$.

Proof Suppose that we are given an election $E=(C, V)$. We can check in polynomialtime if $E \in \mathcal{E}$, and if so, output the consensus winner $\mathcal{W}(E)$ as $\mathcal{R}$ is compatible with $\mathcal{K}$.

Otherwise, for each candidate $c \in C$, we can construct in polynomial time an election $E_{c}=\left(C, V_{c}\right)$, where $E_{c} \in \mathcal{E}$ and $\mathcal{W}\left(E_{c}\right)=c$. Now, $c \in \mathcal{R}(E)$ if and only if $d\left(E, E_{c}\right)=1$. Thus we can query the distance oracle $d$ on $|C|$ inputs of the form $\left(E, E_{c}\right)$ and output the set $\left\{c \mid d\left(E, E_{c}\right)=1\right\}$ which will be exactly $\mathcal{R}(E)$.

For example, Proposition 3 implies that, if $\mathrm{P} \neq \mathrm{NP}$, the distance produced in the proof of Theorem 3 for the rationalization of Kemeny rule with respect to $\mathcal{S}$ is not 
polynomial-time computable. On the other hand, we know that Kemeny does have a very natural rationalization with respect to $\mathcal{S}$ via distance $\widehat{d}_{\text {swap. }}$. This shows once more that it is not the fact of distance-rationalizability itself that is interesting but the distances and consensus classes used in this distance-rationalization.

The requirement that the distance should be polynomial-time computable is essential for the distance rationalizability framework to be interesting. We will impose this condition in addition to further, structural, restriction on the distances that we will introduce in the next section.

\section{Distance-Rationalizability via Votewise Distances}

The results of previous section make it clear that we need to restrict the set of distances that we consider acceptable to use in distance-rationalizations. To identify an appropriate restriction, consider the constructions used to rationalize Borda, Plurality and Litvak rules via distances $\widehat{d}_{\text {swap }}, \widehat{d}_{\text {discr }}$ and $\widehat{d}_{\text {spear }}$, respectively (see Section 2 ). To build either of these distances, we first defined a distance over votes and then extended it to a distance over elections (with the same candidate sets and equal-cardinality voter lists) via summing the distances between respective votes. This technique can be interpreted as taking the direct product of the metric spaces that correspond to individual votes, and defining the distance on the resulting space via the $\ell_{1}$-norm. It turns out that distances obtained in this manner (possibly using norms other than $\ell_{1}$ ), which we will call votewise distances, are very versatile and expressive. They are also attractive from the social choice point of view, as they exhibit continuous and monotone dependence of the distance on the voters' opinions.

In this section we will define votewise distances rigorously and attempt to answer the first two of the following three important questions regarding voting rules that can be rationalized via votewise distances:

(a) What properties do such rules have?

(b) Which rules can be rationalized with respect to votewise distances?

(c) What is the complexity of winner determination for such rules?

The last question will be discussed in Section 5 .

4.1 Definition and General Properties of Votewise Rules

Definition 4 Given a vector space $S$ over $\mathbb{R}$, a norm on $S$ is a mapping $N$ from $S$ to $\mathbb{R}$ that satisfies the following properties:

(i) positive scalability: $N(\alpha u)=|\alpha| N(u)$ for all $u \in S$ and all $\alpha \in \mathbb{R}$;

(ii) positive semidefiniteness: $N(u) \geq 0$ for all $u \in S$, and $N(u)=0$ if and only if $u=0$;

(iii) triangle inequality: $N(u+v) \leq N(u)+N(v)$ for all $u, v \in S$.

A well-known class of norms on $\mathbb{R}^{n}$ are the $p$-norms $\ell_{p}$ given by $\ell_{p}\left(x_{1}, \ldots, x_{n}\right)=$ $\left(\sum_{i=1}^{n}\left(\left|x_{i}\right|^{p}\right)\right)^{\frac{1}{p}}$, with the convention that $\ell_{\infty}\left(x_{1}, \ldots, x_{n}\right)=\max \left\{x_{1}, \ldots, x_{n}\right\}$. Hereafter by $S_{n}$ we denote the group of permutations on $\{1, \ldots, n\}$. A norm $N$ on $\mathbb{R}^{n}$ is said to be symmetric if it satisfies $N\left(x_{1}, \ldots, x_{n}\right)=N\left(x_{\sigma(1)}, \ldots, x_{\sigma(n)}\right)$ for any permutation $\sigma \in S_{n}$; clearly, all $p$-norms are symmetric. We can now define our family of votewise distances. 
Definition 5 We say that a function $d$ on pairs of preference profiles is votewise if the following conditions hold:

1. $d\left(E, E^{\prime}\right)=+\infty$ if $E$ and $E^{\prime}$ have a different set of candidates or a different number of voters.

2. For any set of candidates $C$, there exists a distance $d_{C}$ defined on votes over $C$;

3. For any $n \in \mathbb{N}$, there exists a norm $N_{n}$ on $\mathbb{R}^{n}$ such that for any two preference profiles $E=(C, U), E^{\prime}=(C, V)$ with $U=\left(u_{1}, \ldots, u_{n}\right)$ and $V=\left(v_{1}, \ldots, v_{n}\right)$ we have $d\left(E, E^{\prime}\right)=N_{n}\left(d_{C}\left(u_{1}, v_{1}\right), \ldots, d_{C}\left(u_{n}, v_{n}\right)\right)$.

It is well known that any function defined in this manner is a metric. Thus, in what follows, we refer to votewise functions as votewise distances; we will also use the term " $N$-votewise distance" to refer to a votewise distance defined via a norm $N$, and denote a votewise distance on the set of profiles that is based on a distance $d$ over votes by $\widehat{d}$. Similarly, we will use the term $N$-votewise rules to refer to voting rules that can be distance-rationalized via one of our four consensus classes and an $N$-votewise distance.

An important special case of our framework is when $N_{n}$ is the $\ell_{1}$-norm, i.e., $N_{n}\left(x_{1}, \ldots, x_{n}\right)=\left|x_{1}\right|+\cdots+\left|x_{n}\right|$; we will call any such distance an additively votewise distance, or, in line with the notation introduced above, an $\ell_{1}$-votewise distance. Although $\ell_{1}$-votewise distances were the only votewise distances used in distance rationalizability constructions of social choice rules, $\ell_{2}$-metric has been used in aggregation of expert opinions, e.g., in Litvak (1983). Also, $N$-votewise distances with $N \neq \ell_{1}$ are almost as easy to work with as $\ell_{1}$-votewise distances and may be useful for rationalizing natural voting rules in the future. In fact, later on we will see that simplified Bucklin and the Threshold rules are an $\ell_{\infty}$-votewise.

In this section we consider several basic properties of voting rules. Specifically, given a consensus class $\mathcal{K}$ and a votewise distance $\widehat{d}$, we ask under which circumstances a $(\mathcal{K}, \widehat{d})$-rationalizable voting rule is anonymous, neutral, or consistent. To start, we recall the formal definitions of these properties.

Let $E=(C, V)$ be an election with $V=\left(v_{1}, \ldots, v_{n}\right)$, and $C=\left(c_{1}, \ldots, c_{m}\right)$ where we view here the set of candidates as a list too. Let $\sigma$ and $\pi$ be permutations of $V$ and $C$, respectively. For any $C^{\prime} \subseteq C$, set $\pi\left(C^{\prime}\right)=\left\{\pi(c) \mid c \in C^{\prime}\right\}$. Let $\tilde{\pi}(v)$ be the vote obtained from $v$ by replacing each occurrence of a candidate $c \in C$ by an occurrence of $\pi(c)$; we can extend this definition to preference profiles by setting $\tilde{\pi}\left(v_{1}, \ldots, v_{n}\right)=\left(\tilde{\pi}\left(v_{1}\right), \ldots, \tilde{\pi}\left(v_{n}\right)\right)$.

Anonymity. A voting rule is anonymous if its result depends only on the number of voters reporting each preference order. Formally, a voting rule $\mathcal{R}$ is anonymous if for every election $E=(C, V)$ with $V=\left(v_{1}, \ldots, v_{n}\right)$ and every permutation $\sigma$ of $V$, the election $E^{\prime}=(C, \sigma(V))$ satisfies $\mathcal{R}(E)=\mathcal{R}\left(E^{\prime}\right)$.

Neutrality. A voting rule is neutral if its result does not depend on the candidates' names. Formally, a voting rule $\mathcal{R}$ is neutral if for every election $E=(C, V)$ and every permutation $\pi$ of $C$, the election $E^{\prime}=(C, \tilde{\pi}(V))$ satisfies $\mathcal{R}(E)=\pi^{-1}\left(\mathcal{R}\left(E^{\prime}\right)\right)$.

Consistency. A voting rule $\mathcal{R}$ is consistent if for any two elections $E_{1}=\left(C, V_{1}\right)$ and $E_{2}=\left(C, V_{2}\right)$ such that $\mathcal{R}\left(E_{1}\right) \cap \mathcal{R}\left(E_{2}\right) \neq \emptyset$, the election $E=\left(C, V_{1}+V_{2}\right)$ (i.e., the election where the collections of voters from $E_{1}$ and $E_{2}$ are concatenated) satisfies $\mathcal{R}(E)=\mathcal{R}\left(E_{1}\right) \cap \mathcal{R}\left(E_{2}\right)$. This property was introduced by Young (1975) and is also known as reinforcement (Chebotarev \& Shamis, 1998). 
Homogeneity. A voting rule $\mathcal{R}$ is homogeneous if for every election $E=(C, V)$ and every positive integer $k$ it holds that $\mathcal{R}(E)=\mathcal{R}(k E)$, where $k E$ is the election $k E=(C, k V)$. This property is weaker than consistency.

Continuity. A voting rule $\mathcal{R}$ is continuous if for any two elections $E_{1}=\left(C, V_{1}\right)$ and $E_{2}=\left(C, V_{2}\right)$ such that $\mathcal{R}\left(E_{1}\right)=\{a\}$, the election $E=\left(C, n V_{1}+V_{2}\right)$ also satisfies $\mathcal{R}(E)=\{a\}$ for sufficiently large $n$. This property was introduced by Young (1975).

Monotonicity. A voting rule $\mathcal{R}$ is monotone if for every election $E=(C, V)$ and every $c \in \mathcal{R}(E)$ it holds that if a voters change her minds about $c$ moving $c$ higher in her ranking (but do not change relative positions of other alternatives) then $c$ remains a winner of the new election. In Moulin's (1991) book this property is called ceteris paribus monotonicity to distinguish it from Maskin monotonicity.

If $\mathcal{K}=(\mathcal{E}, \mathcal{W})$ is a consensus class, then, of course, these definitions are also applicable to $\mathcal{W}$ if $\mathcal{E}$ is closed under the respective profile transformations. For example, we will say that $\mathcal{K}$ is anonymous if for any profile $U$ from $\mathcal{E}$ any profile $U^{\prime}$ obtained by permuting linear orders in $U$ is also in $\mathcal{E}$ and $\mathcal{W}(U)=\mathcal{W}\left(U^{\prime}\right)$.

We will start with showing that for votewise distance-rationalizable rules, a symmetric norm produces an anonymous rule.

Proposition 4 Suppose that $\mathcal{K}$ is an anonymous consensus class and a voting rule $\mathcal{R}$ is $(\mathcal{K}, \widehat{d})$-rationalizable, where $\widehat{d}$ is an $N$-votewise distance for a symmetric norm $N$ and $d$ is a pseudodistance over votes. Then $\mathcal{R}$ is anonymous.

Proof Let $E_{V}=(C, V)$, where $|V|=n$, be an election, and let $\sigma$ be a permutation of $V$. Fix a candidate $c \in \mathcal{R}(E)$, and let $E_{U}=(C, U)$ be a $\mathcal{K}$-consensus election that is closest to $E$ among those whose winner is $c$. We form elections $E_{V}^{\prime}=\left(C, V^{\prime}\right)$ and $E_{U}^{\prime}=\left(C, U^{\prime}\right)$ by setting $V^{\prime}=\sigma(V)$ and $U^{\prime}=\sigma(U)$. As $\mathcal{K}$ is anonymous the election $E_{U}^{\prime}$ is a $\mathcal{K}$-consensus again, and, moreover, $\widehat{d}\left(E_{V}, E_{U}\right)=\widehat{d}\left(E_{V}^{\prime}, E_{U}^{\prime}\right)$. Now, suppose that there exists a $\mathcal{K}$-consensus election $E_{W}=(C, W)$ such that $\widehat{d}\left(E_{V}^{\prime}, E_{W}\right)<\widehat{d}\left(E_{V}^{\prime}, E_{U}^{\prime}\right)$. Then for the election $E_{W}^{\prime}=\left(C, \sigma^{-1}(W)\right)$ we have

$$
\widehat{d}\left(E_{V}, E_{W}^{\prime}\right)=\widehat{d}\left(E_{V}^{\prime}, E_{W}\right)<\widehat{d}\left(E_{V}^{\prime}, E_{U}^{\prime}\right)=\widehat{d}\left(E_{V}, E_{U}\right)
$$

a contradiction with our choice of $E_{U}$. Thus, any winner of $E_{V}$ is a winner of $E_{V}^{\prime}$. By considering permutation $\sigma^{-1}$, we also obtain that any winner of $E_{V}^{\prime}$ is a winner of $E_{V}$ too.

We have shown that for votewise rules, anonymity is essentially a property of the underlying norm. In contrast, neutrality is inherited from the underlying distance over votes.

Definition 6 Let $C$ be a set of candidates and let $d$ be a distance on votes over $C$. We say that $d$ is neutral if for each permutations $\pi$ over $C$ and any two votes $u$ and $v$ over $C$ it holds that $d(u, v)=d(\tilde{\pi}(u), \tilde{\pi}(v))$.

Proposition 5 Suppose that a voting rule $\mathcal{R}$ is $(\mathcal{K}, \widehat{d})$-rationalizable for a neutral consensus class $\mathcal{K}$ and a neutral pseudodistance $d$ over votes. Then $\mathcal{R}$ is neutral.

Proof The proof is similar to the proof of Proposition 4 and we omit it. 
It is natural to ask if the converse of Proposition 5 is also true, i.e., if every neutral votewise rule can be rationalized via a neutral distance. Indeed, paper of Conitzer et al. (2009) provides a positive answer to a similar question in the context of representing voting rules as maximum likelihood estimators. However, it is not clear how their approach can be adapted to work in our setting. Nevertheless, all votewise distances that have so far arisen in the study of distance rationalizability of common voting rules are neutral.

Our results for anonymity and neutrality are applicable to all consensus classes considered in this paper. In contrast, when discussing consistency, we need to limit ourselves to the unanimity consensus, and to $\ell_{p}$-votewise rules.

Theorem 4 Suppose that a voting rule $\mathcal{R}$ is $\left(\mathcal{U}, \widehat{d^{p}}\right)$-rationalizable for some pseudodistance $d$ over votes. Then $\mathcal{R}$ is consistent.

Proof Let $E_{1}=\left(C, V_{1}\right)$ and $E_{2}=\left(C, V_{2}\right)$ be two elections over the same candidate set $C$ such that $\mathcal{R}\left(E_{1}\right) \cap \mathcal{R}\left(E_{2}\right) \neq \emptyset$, and let $E=\left(C, V_{1}+V_{2}\right)$. First, we will show that $\mathcal{R}\left(E_{1}\right) \cap \mathcal{R}\left(E_{2}\right) \subseteq \mathcal{R}(E)$. Fix a candidate $c \in \mathcal{R}\left(E_{1}\right) \cap \mathcal{R}\left(E_{2}\right)$. By definition, there are two unanimous profiles, $U_{1}$ and $U_{2}$, such that $c$ is the unanimity winner of $U_{i}$ and $U_{i} \in \arg \min _{X \in \mathcal{U}} \widehat{d}\left(V_{i}, X\right)$ for $i=1,2$.

For the sake of contradiction, suppose that $c \notin \mathcal{R}(E)$. Clearly, $U_{1}+U_{2}$ is a unanimous profile with winner $c$. As $c \notin \mathcal{R}(E)$, there must be another unanimous profile $W_{1}+W_{2}$ with $\left|W_{1}\right|=\left|V_{1}\right|,\left|W_{2}\right|=\left|V_{2}\right|$ such that

$$
\widehat{d}\left(V_{1}+V_{2}, U_{1}+U_{2}\right)>\widehat{d}\left(V_{1}+V_{2}, W_{1}+W_{2}\right) .
$$

Since $\widehat{d}$ is an $\ell_{p}$-votewise distance, this inequality is equivalent to

$$
\sqrt[p]{\widehat{d}\left(V_{1}, U_{1}\right)^{p}+\widehat{d}\left(V_{2}, U_{2}\right)^{p}}>\sqrt[p]{\widehat{d}\left(V_{1}, W_{1}\right)^{p}+\widehat{d}\left(V_{2}, W_{2}\right)^{p}}
$$

However, by the choice of $U_{1}$ and $U_{2}$, it holds that

$$
\widehat{d}\left(V_{1}, U_{1}\right) \leq \widehat{d}\left(V_{1}, W_{1}\right), \quad \widehat{d}\left(V_{2}, U_{2}\right) \leq \widehat{d}\left(V_{2}, W_{2}\right)
$$

which immediately yields a contradiction with (6), and so $c \in \mathcal{R}(E)$.

To show that $\mathcal{R}(E) \subseteq \mathcal{R}\left(E_{1}\right) \cap \mathcal{R}\left(E_{2}\right)$, consider a $c^{\prime} \in \mathcal{R}(E)$. Since $c$ and $c^{\prime}$ are both in $\mathcal{R}(E)$, there exists a unanimous profile $X_{1}+X_{2}$ with winner $c^{\prime}$ such that $\left|X_{1}\right|=\left|V_{1}\right|,\left|X_{2}\right|=\left|V_{2}\right|$ and $\widehat{d}\left(V_{1}+V_{2}, X_{1}+X_{2}\right)=\widehat{d}\left(V_{1}+V_{2}, U_{1}+U_{2}\right)$. that is,

$$
\sqrt[p]{\widehat{d}\left(V_{1}, X_{1}\right)^{p}+\widehat{d}\left(V_{2}, X_{2}\right)^{p}}=\sqrt[p]{\widehat{d}\left(V_{1}, U_{1}\right)^{p}+\widehat{d}\left(V_{2}, U_{2}\right)^{p}} .
$$

On the other hand, we have

$$
\widehat{d}\left(V_{1}, U_{1}\right) \leq \widehat{d}\left(V_{1}, X_{1}\right), \quad \widehat{d}\left(V_{2}, U_{2}\right) \leq \widehat{d}\left(V_{2}, X_{2}\right),
$$

It follows from (7) that both of the inequalities above are, in fact, equalities. Thus, by our choice of $U_{1}$ and $U_{2}$, for $i=1,2$, we obtain that $X_{i} \in \arg \min _{X \in \mathcal{U}} \widehat{d}\left(V_{i}, X\right)$. Since $c^{\prime}$ is the unanimity winner in $X_{1}$ and $X_{2}$, it follows that $c^{\prime} \in \mathcal{R}\left(E_{1}\right) \cap \mathcal{R}\left(E_{2}\right)$. 
For consensus classes $\mathcal{S}$ and $\mathcal{C}$ the analogue of Theorem 4 cannot be proved. Counterexamples are provided by the Kemeny rule and the Dodgson rule, respectively. Both are rationalizable via $\widehat{d}_{\text {swap }}$ distance. However, since both extend Condorcet rule, by Theorem 2 of Young and Levenglick (1978), they cannot be consistent and Dodgson rule is not even homogeneous (Brandt, 2009).

Note that the second part of the proof of Theorem 4 does not go through for $\ell_{\infty}$ votewise rules so we can claim only $\mathcal{R}\left(E_{1}\right) \cap \mathcal{R}\left(E_{2}\right) \subseteq \mathcal{R}(E)$. However if $E_{1}=E_{2}$ the argument does go through. This means that all $\ell_{\infty}$-votewise rules distance rationalized relative to the unanimity consensus are homogeneous. Also it is easy to see that when $E_{1}=E_{2}$ the argument also work with the strong unanimity consensus $\mathcal{S}$. Thus we obtain

Theorem 5 Suppose that voting rule $\mathcal{R}$ is $\left(\mathcal{K}, \widehat{d^{p}}\right)$-rationalizable, where $\mathcal{K} \in\{\mathcal{S}, \mathcal{U}\}$, $p \in \mathbb{N} \cup\{\infty\}$, and $d$ is a pseudodistance over votes. Then $\mathcal{R}$ is homogeneous.

In general $\ell_{\infty}$-votewise distance-rationalizable rules are very prone to being homogeneous: we need to impose only a very mild condition on the consensus class $\mathcal{K}$.

Definition 7 Let $\mathcal{K}=(\mathcal{E}, \mathcal{W})$ be a consensus class. We say that it is split-homogeneous if the following two conditions hold:

(a) If $U \in \mathcal{E}$ is a consensus profile then $n U$ is a consensus profile too with $\mathcal{W}(U)=$ $\mathcal{W}(n U)$

(b) If $U$ and $W$ are two profiles, with $n$ votes each, such that $U+W \in \mathcal{E}$, then either $U \in \mathcal{E}$ or $W \in \mathcal{E}$.

Any known consensus class but $\mathcal{C}$ are of this nature (see the counterexample for $\mathcal{C}$ in the appendix).

It turns out that combining a split-homogeneous consensus class with an $\ell_{\infty^{-}}$ votewise distance produces a homogeneous rule.

Theorem 6 Let $C$ be any set of alternatives and d be any pseudodistance on $P(C)$. Let $\mathcal{K}=(\mathcal{E}, \mathcal{W})$ be a split-homogeneous consensus class. Then any $\left(\mathcal{K}, \widehat{d^{\infty}}\right)$-rationalizable voting rule $\mathcal{R}$ is homogeneous.

Proof We will prove that for any election $E=(C, V)$ we have $\mathcal{R}(E)=\mathcal{R}(2 E)$, the general case is similar. Let $c$ be a winner of $E$ and $U$ be the closest consensus profile to $V$ with $\mathcal{W}(U)=c$. Then

$$
k=\widehat{d^{\infty}}(V, U) \leq \widehat{d^{\infty}}\left(V, U^{\prime}\right) \text { for any } U^{\prime} \in \mathcal{E} .
$$

Due to the nature of $\ell_{\infty}$-metric we have

$$
\widehat{d^{\infty}}(2 V, 2 U)=\widehat{d^{\infty}}(V, U)=k
$$

and $2 U$ is a consensus profile by condition (a). Suppose that $c \in \mathcal{W}(U)=\mathcal{W}(2 U)$ is not a winner of $2 E$. Then there exist a profile $X+Y \in \mathcal{E}$, each with $n$ votes, such that $\widehat{d^{\infty}}(2 V, X+Y)<k$. Since our distance is an $\ell_{\infty}$ one, we have

$$
\widehat{d^{\infty}}(V, X)<k \quad \text { and } \quad \widehat{d^{\infty}}(V, Y)<k .
$$

However the consensus class is split-homogeneous so either $X \in \mathcal{E}$ or $Y \in \mathcal{E}$ which contradicts to (8) and (9). 
Corollary 1 Bucklin and Threshold rules are homogeneous.

Both of these two rules however are not consistent. For $\ell_{p}$-votewise rules and even for additively votewise rules Theorem 6 does not hold. In particular, in the next section we will see that $\mathcal{M}$-Borda, which will be defined there, is not homogeneous.

The condition of split-homogeneity in Theorem 6 is only sufficient but not necessary. Let us define the following voting rule.

Definition 8 The rule which always selects $\left(\mathcal{C}, d_{\text {swap }}^{\infty}\right)$-winners will be called Dodgson ${ }^{\infty}$.

This min-max version of the Dodgson's rule is remarkable in several ways. In particular, it is homogeneous (and Dodgson's rule is not!).

Proposition 6 Dodgson $^{\infty}$ is homogeneous.

Proof The Dodgson ${ }^{\infty}$ 's score score ${ }_{\text {Dodg }}^{\infty}(V)$ of the alternative $c \in C$ in a profile $V$ is the smallest positive integer $k$ such it is possible to make $c$ a Condorcet winner by making no more than $k$ swaps in each preference order of $V$. The winner is the alternative with the lowest score. The statement follows from the fact that if $c$ is a Dodgson ${ }^{\infty}$ 's winner of $s V$ for some positive integer $s$ with the score $k$, then there exist a sequence of swaps that

- makes $c$ a Condorcet winner;

- makes no more than $k$ swaps in any of the preference orders and exactly $k$ in some of them;

- makes exactly the same swaps in each of $s$ copies of $V$.

This means score ${ }_{\text {Dodg }}^{\infty}(s V)=\operatorname{score}_{\text {Dodg }}^{\infty}(V)$ and this proves the statement.

Now let us deal with the continuity.

Proposition 7 Suppose that a voting rule $\mathcal{R}$ is $\left(\mathcal{U}, \widehat{d}^{p}\right)$-rationalizable, where $d$ is a pseudodistance over votes. Then $\mathcal{R}$ is continuous.

Proof Let $E_{1}=\left(C, V_{1}\right)$ and $E_{2}=\left(C, V_{2}\right)$ be two elections over the same candidate set $C$ such that $\mathcal{R}\left(E_{1}\right)=a$, and let $E_{n}=\left(C, n V_{1}+V_{2}\right)$. By definition, there are two unanimous profiles, $U_{1}$ and $U_{2}$, such that $a$ is the unanimity winner of $U_{1}, c \neq a$ is the unanimity winner of $U_{2}$ and $U_{i} \in \arg \min _{X \in \mathcal{U}} \widehat{d}\left(V_{i}, X\right)$ for $i=1,2$. The distance from any $X \in \mathcal{U}$ to $V_{n}=n V_{1}+V_{2}$ will be measured as

$$
\widehat{d}\left(n V_{1}+V_{2}, X\right)=\sqrt[p]{n^{p} \widehat{d}\left(V_{1}, X\right)^{p}+\widehat{d}\left(V_{2}, X\right)^{p}}
$$

For large $n$ this expression is minimized if and only if $\widehat{d}\left(V_{1}, X\right)$ is minimized, so the top alternative of $X$ must be in $\mathcal{R}\left(E_{1}\right)$ and hence $a$. Thus $\mathcal{R}\left(E_{n}\right)=a$ for such $n$.

\section{$4.2 \boldsymbol{\ell}_{\boldsymbol{p}}$-Votewise Rules}

Now that we know that $\ell_{p}$-votewise rules have some desirable properties, let us see which voting rules are in fact $\ell_{p}$-votewise distance rationalizable. We will generally focus on $\ell_{p}$-votewise rules, but we will look at $\ell_{\infty}$ as well. Naturally, we expect the answer to this question to strongly depend on the consensus notion used. Thus, let us consider unanimity, strong unanimity, majority, and Condorcet consensuses one by one.

We start with the unanimity consensus. 
Theorem 7 Let $C$ be a fixed set of $m$ alternatives, $\mathcal{R}$ be a voting rule and $p$ is a positive integer. Suppose $\mathcal{R}$ is $\left(\mathcal{U}, \widehat{d^{p}}\right)$-rationalizable where $d$ is a neutral pseudodistance over votes. Then $\mathcal{R}$ is a scoring rule, that is $\mathcal{R}_{\alpha}$ for some vector $\alpha=\left(\alpha_{1}, \ldots, \alpha_{m}\right){ }^{6}$

Proof By combining Propositions 4, 5 and 7 and Theorem 4 we conclude that any rule that is $\left(\mathcal{U}, \widehat{d^{p}}\right)$-rationalizable, where $d$ is a neutral pseudodistance, is neutral, anonymous, consistent and continuous and by Young's famous characterization result (Young, 1975) it is a (simple) scoring rule.

This leads to a surprising conclusion that for unanimous consensus we cannot get anything new by using $\ell_{p}$-votewise distances with $p>1$. This probably explains why we have never heard about, for example, quadratic Borda rule where the score is calculated by taking squares of respective weights of alternatives and not weights themselves.

For additive votewise distances we can formulate a stronger statement.

Theorem 8 Let $\mathcal{R}$ be a voting rule. There exists a neutral pseudodistance $d$ such that $\mathcal{R}$ is $\left(\mathcal{U}, \widehat{d^{1}}\right)$-rationalizable if and only if $\mathcal{R}$ is a scoring rule.

Proof The "if" direction was essentially shown by us; it is not hard to see that the distance used in the proof of Theorem 1 is a neutral $\ell_{1}$-votewise pseudodistance. The "only if" direction follows from Theorem 7.

Here we have an if and only if statement. Theorem 7 goes only one way since we have no idea how to obtain an $\ell_{p}$-votewise rationalizations for scoring rules similar to the one in Theorem 1 for any $p>1$.

Later in Section 5 we will show that we can actually "extract" the weights of the scoring rule from the corresponding distance, albeit not efficiently (see Section 5 for a discussion of the related complexity issues).

Theorem 8 gives a complete characterization of voting rules rationalizable via neutral $\ell_{1}$-votewise distances with respect to the unanimity consensus. However, the situation with respect to other consensus notions is more difficult to describe.

Let us consider strong unanimity next. Intuitively, strong unanimity is quite challenging to work with as it provides very little flexibility. Meskanen and Nurmi (2008) have shown that Kemeny is $\ell_{1}$-votewise with respect to $\mathcal{S}$, but, at least at the first glance, it seems that no other natural rule is. Interestingly, and very counterintuitively, Plurality is also $\ell_{1}$-votewise with respect to strong unanimity while Borda is not. As the proof of these statements are technical we relegate them to the appendix.

Theorem 9 There exists an $\ell_{1}$-votewise distance $\widehat{d}$ such that Plurality rule is $(\mathcal{S}, \widehat{d})$ rationalizable but there is no $\ell_{1}$-votewise distance $\widehat{d}$ such that Borda rule is $(\mathcal{S}, \widehat{d})$-rationalizable.

Thus, the class of rules $\ell_{1}$-votewise rationalizable with respect to $\mathcal{S}$ is rather enigmatic. On the one hand, it does contain Kemeny, a very complex rule, and Plurality, a very simple rule, yet it does not contain other natural scoring rules such as Borda. One

6 Note that here, following Young (1975), we do not require $\alpha_{1}, \ldots, \alpha_{m}$ to be nondecreasing or integer. Indeed, the distance rationalizability framework does not impose any ordering over different positions in a vote, so it works equally well for a scoring rule with any nonnegative $\alpha$. In the Section 4.5 we will see which condition on the distance leads to the ordered $\alpha$. 
characterization of this class is however obtained further in Theorem 23, where this class of rules is shown to be MLERIV for one particular model of noise. This will be proved in Section 6.

For the case of $\mathcal{M}$ some old rules get new rationalizations. For example, the same logic as in Nitzan $(1981)$ tells us that Plurality is $\left(\mathcal{M}, \widehat{d_{\text {discr }}}\right)$-rationalizable.

If combining $\widehat{d}_{\text {discr }}$ with $\mathcal{M}$ gives the same effect as combining it with $\mathcal{U}$, then perhaps the same trick works for $\widehat{d}_{\text {swap }}$, and $\ell_{1}$-votewise $\left(\mathcal{M}, \widehat{d_{\text {swap }}}\right)$-rationalizable rule is Borda? It is easy to see that this is not the case, but the resulting rule is indeed very similar to Borda.

Let us consider the voting rule $\mathcal{R}$ that always selects the set of $\left(\mathcal{M}, \widehat{d_{\text {swap }}}\right)$-winners. Let $E=(C, V)$ be an election, where $C=\left\{c_{1}, \ldots, c_{m}\right\}$ and $V=\left(v_{1}, \ldots, v_{n}\right)$. For each candidate $c_{i} \in C$, let $M\left(c_{i}\right)$ be the multiset of Borda scores $\left\{m-\operatorname{pos}_{v_{j}}\left(c_{i}\right) \mid 1 \leq j \leq n\right\}$. We claim that $\mathcal{R}$ always selects a candidate $c_{i} \in C$ with the highest score calculated as the sum of the highest $\left\lfloor\frac{n}{2}\right\rfloor+1$ values from $M\left(c_{i}\right)$. Indeed, to keep the number of swaps to the consensus to a minimum we have to choose $\left\lfloor\frac{n}{2}\right\rfloor+1$ voters who rank $c_{j}$ highest and move $c_{j}$ to the top position in those. It is easy to see that $\mathcal{R}$ is different from Borda in which case of the score of $c_{i}$ is calculated as the sum of all the values from $M\left(c_{i}\right)$. We will refer to this new rule $\mathcal{R}$ as $\mathcal{M}$-Borda. Not only Borda but in fact, all scoring rules have their " $\mathcal{M}$-variants."

Definition 9 Let $\alpha=\left(\alpha_{1}, \ldots, \alpha_{m}\right)$ be a scoring vector. Voting rule $\mathcal{M}-\mathcal{R}_{\alpha}$ is defined as follows. Let $E=(C, V)$ be an election where $C=\left\{c_{1}, \ldots, c_{m}\right\}$ and $V=\left(v_{1}, \ldots, v_{n}\right)$. For each candidate $c_{i} \in C$, let $M\left(c_{i}\right)$ be the multiset of numbers $\left\{\alpha_{\text {pos }_{v_{j}}\left(c_{i}\right)} \mid 1 \leq j \leq\right.$ $n\}$. The $\mathcal{M}-\mathcal{R}_{\alpha}$ score of candidate $c_{i}$ is the sum of the $\left\lfloor\frac{n}{2}\right\rfloor+1$ highest values from $M\left(c_{i}\right)$. The candidates with the highest $\mathcal{M}-\mathcal{R}_{\alpha}$ scores are the winners.

We will refer to voting rules from Definition 9 as $\mathcal{M}$-scoring rules. The next theorem (preceded by a technical lemma) is a characterization of $\mathcal{M}$-scoring rules analogous to Theorem 8.

By $P(C, c)$ we denote the set of linear orders over the set of alternatives $C$ whose top alternative is $c$.

Lemma 1 Let $\mathcal{R}$ be a voting rule that is $(\mathcal{M}, \widehat{d})$-rationalized. Let $E=(C, V)$ be an arbitrary election where $V=\left(v_{1}, \ldots, v_{n}\right)$ and let $(C, U)$ be an $\mathcal{M}$-consensus such that $\widehat{d}(U, V)$ is minimal among all $\mathcal{M}$-consensuses over $C$ with $n$ votes. Let $c \in$ $C$ be the consensus winner of $(C, U)$. Then, for each $i, 1 \leq i \leq n$, either $u_{i} \in$ $\arg \min _{x \in P(C, c)} d\left(x, v_{i}\right)$ or $u_{i}=v_{i}$ (or both).

Proof Follows directly from the definitions of $\mathcal{M}$ and $\ell_{1}$-votewise distances. It can be explained as follows. Given a profile $V$ we have to find the closest $\mathcal{M}$-consensus $U$ to it. For this we will have to modify at most $\left\lfloor\frac{n}{2}\right\rfloor+1$ votes in $V$ so for the rest of the votes we will have $u_{i}=v_{i}$. When we modify the remaining votes we do it optimally, hence $u_{i} \in \arg \min _{x \in P(C, c)} d\left(x, v_{i}\right)$.

Theorem 10 For each scoring vector $\alpha=\left(\alpha_{1}, \ldots, \alpha_{m}\right)$ and each election $E=(C, V)$ with $|C|=m$, a candidate $c_{j} \in C$ is a winner of $E$ according to $\mathcal{M}-\mathcal{R}_{\alpha}$ if and only if $c_{j}$ is a $\left(\mathcal{M}, \widehat{d_{\alpha}}\right)$-winner of $E$.

Proof Goes along the same lines as the proof of Theorem 1 with the use of Lemma 1 in appropriate places. 
Theorem 11 Let $\mathcal{R}$ be a voting rule. There exists a neutral pseudodistance $d$ over votes such that $\mathcal{R}$ is $(\mathcal{M}, \widehat{d})$-rationalizable if and only if $\mathcal{R}=\mathcal{M}-\mathcal{R}_{\alpha}$ for some scoring vector $\alpha$.

Proof The "if" direction is proved in Theorem 10 since the pseudodistance $\widehat{d_{\alpha}}$ is neutral and $\ell_{1}$-votewise. For the "only if" direction, let $\mathcal{R}$ be a $(\mathcal{U}, \widehat{d})$-rationalizable voting rule, where $\widehat{d}$ is a neutral, $\ell_{1}$-votewise pseudodistance based on a pseudodistance $d$. We will now show how to derive a scoring rule $\mathcal{M}-\mathcal{R}_{\left(\alpha_{1}, \ldots, \alpha_{m}\right)}$ that corresponds to $\mathcal{R}$ for $m$ candidates.

Let $C=\left\{c_{1}, \ldots, c_{m}\right\}$, and consider an arbitrary preference profile $V=\left(v_{1}, \ldots, v_{n}\right)$ over $C$. Fix any vote $v \in V$ and let $c \in C$ be such that $\operatorname{pos}_{v}(c)=k$. Let also $u$ be the nearest vote to $v$ that ranks $c$ first. Define $\beta_{k}=d(u, v)$. Note that by neutrality the value of $\beta_{k}$ is independent of the choice of $v$. Now, consider a candidate $c$ that is ranked in position $t_{i}$ in $v_{i}$ for $i=1, \ldots, n$. Clearly, the distance from $V$ to the nearest profile in $\mathcal{M}$ in which $c$ wins is given by the sum of $\left\lfloor\frac{n}{2}\right\rfloor+1$ lowest values in the multiset $\left\{\beta_{i} \mid 1 \leq i \leq n\right\}$. Thus, to transform the vector $\beta=\left(\beta_{1}, \ldots, \beta_{m}\right)$ into a score vector, we need to "reverse" it by setting $\alpha_{j}=B-\beta_{j}$ for $j=1, \ldots, m$ where $B$ is large enough, e.g., $B=\max _{j=1}^{m} \beta_{j}$. It is immediate that $\mathcal{M}-\mathcal{R}_{\left(\alpha_{1}, \ldots, \alpha_{m}\right)}$ is exactly $\mathcal{R}$ for $m$ candidates.

\section{$4.3 \ell_{\infty}$-Votewise Rules}

The characterizations in the previous subsection of neutral $\ell_{p}$-votewise rules strictly excluded $\ell_{\infty}$-votewise rules, which as we saw can be substantially different. They are nevertheless important as several important rules have distance rationalizations relative to $\ell_{\infty}$-votewise distances.

Theorem 12 The threshold rule is $\left(\mathcal{U}, \widehat{d_{\text {borda }}^{\infty}}\right)$-rationalizable.

Proof Follows directly from definitions.

More interestingly, we can show that simplified Bucklin is $\ell_{\infty}$-votewise with respect to $\mathcal{M}$.

Theorem 13 Simplified Bucklin is both $\left(\mathcal{M}, \widehat{d_{\text {sert }}^{\infty}}\right)$-rationalizable and $\left(\mathcal{M}, \widehat{d_{\text {spear }}^{\infty}}\right)$-rationalizable.

Proof Let $E=(C, V)$ be an election with $V=\left(v_{1}, \ldots, v_{n}\right)$, and let $c$ be a candidate in $C$. Let $k$ be the smallest integer such that $c$ is a $k$-majority winner.

Now consider an arbitrary election $E_{U}=(C, U), U=\left(u_{1}, \ldots, u_{n}\right)$, in which $c$ is a strict-majority winner. We have $\widehat{d_{\text {sert }}^{\infty}}\left(E, E_{U}\right) \geq k$. Indeed, suppose $\ell=\left\lfloor\frac{n}{2}\right\rfloor+1$ and suppose without loss of generality that $c$ is placed on the top by the first $\ell$ linear orders in $U$, that is $u_{1}, \ldots, u_{\ell}$. If $\operatorname{pos}_{v_{i}}(c)<k$ for $i=1,2, \ldots, \ell$, then $k$ is not the smallest. This means that for some $1 \leq i \leq \ell$ we have $\operatorname{pos}_{v_{i}}(c) \geq k$. This implies $d_{\text {sert }}\left(u_{i}, v_{i}\right) \geq k$ and $\widehat{d_{\text {sert }}^{\infty}}\left(E, E_{U}\right)=\max _{i=1}^{n} d_{\text {sert }}\left(u_{i}, v_{i}\right) \geq k$ as well.

On the other hand, there is a strict majority consensus $E_{W}=(C, W)$ with winner $c$ such that $\widehat{d_{\text {sert }}^{\infty}}\left(E, E_{W}\right)=k$. Indeed, we can construct $E_{W}$ from $E$ by shifting $c$ to the top in each vote that ranks $c$ among the top $k$ candidates (without changing anything else in those votes). Due to the minimality of $k$ one of them has to be shifted from $k$ th position securing that the distance is at least $k$. 
Thus, for each simplified Bucklin winner $c$ of $E$ there exists an election $E_{W} \in \mathcal{M}$ such that $E_{W} \in \arg \min _{E^{\prime} \in \mathcal{M}} \widehat{d_{\text {sert }}^{\infty}}\left(E, E^{\prime}\right)$. That is, simplified Bucklin is $\left(\mathcal{M}, \widehat{d_{\text {sert }}^{\infty}}\right)$ rationalizable.

The proof of $\left(\mathcal{M}, \widehat{d_{\text {spear }}^{\infty}}\right)$-rationalization is similar.

The full version of Bucklin rule is also rationalizable via a distance very similar to the one for simplified Bucklin but, nonetheless, not votewise. Finding further natural voting rules that are votewise rationalizable with respect to $\mathcal{M}$ is an open question.

Our knowledge of rules that are votewise rationalizable with respect to $\mathcal{C}$ is more limited. Dodgson, according to its definition, is $\ell_{1}$-votewise rationalizable with respect to $\mathcal{C}$, and we can see that no scoring rule is distance-rationalizable with respect to $\mathcal{C}$ because scoring rules are not Condorcet-consistent (Moulin, 1991). It is very interesting if, e.g., Young's rule is votewise with respect to $\mathcal{C}$ (however, see Section 7 for some comments).

\subsection{STV Is Not Votewise Distance-Rationalizable}

We will now prove that STV cannot be rationalized with respect to $\mathcal{S}$ and (almost) any votewise distance. We need the following definition.

Definition 10 (Bauer, Stoer, and Witzgall (1961)) A norm $N$ in $\mathbb{R}^{n}$ is monotonic in the positive orthant, or $\mathbb{R}_{+}^{n}$-monotonic, if for any two vectors $\left(x_{1}, \ldots, x_{n}\right),\left(y_{1}, \ldots, y_{n}\right) \in$ $\mathbb{R}_{+}^{n}$ such that $x_{i} \leq y_{i}$ for all $i=1, \ldots, n$ we have $N\left(x_{1}, \ldots, x_{n}\right) \leq N\left(y_{1}, \ldots, y_{n}\right)$.

Bauer, Stoer, and Witzgall (1961) provide a discussion of norms that are monotonic in the positive orthant. We remark that this is a fairly weak notion of monotonicity: the class of $\mathbb{R}_{+}^{n}$-monotonic norms strictly contains the class of all monotonic norms as defined in Bauer et al (1961). The requirement of $\mathbb{R}_{+}^{n}$-monotonicity is very natural when the norm in question is to be used to construct a product metric, as in our case. For example, $\mathbb{R}_{+}^{n}$-monotonic norms are used in a similar context in non-preemptive goal programming (Carrizosa \& Fliege, 2002).

We say that a votewise distance is monotonic if the respective norm is monotonic in the positive orthant. (Note that the values of the norm outside of the positive orthant are not used to compute the distance.)

Theorem 14 For three candidates, STV (together with any intermediate tie-breaking rule) is not $(\mathcal{S}, \widehat{d})$-rationalizable with respect to any neutral anonymous monotonic votewise distance $\widehat{d}$.

Proof For the sake of contradiction, suppose that STV can be rationalized with respect to $\mathcal{S}$ via a neutral anonymous monotonic votewise distance $\widehat{d}$, and let $N$ denote the corresponding norm. Consider a profile

$$
V=(\underbrace{a b c, \ldots, a b c}_{k}, \underbrace{b c a, \ldots, b c a}_{k}, c a b) .
$$


By neutrality and symmetry $d(a b c, b c a)=d(a b c, c a b)=S, d(a b c, a c b)=T, d(a b c, b a c)=$ $B, d(a b c, c b a)=C$ for some numbers $B, C, S, T$. Hence

$$
\begin{aligned}
d_{1} & =\widehat{d}(V, a b c)=N(0, \ldots, 0, S, \ldots, S, S), \\
d_{2} & =\widehat{d}(V, a c b)=N(T, \ldots, T, C, \ldots, C, B), \\
d_{3} & =\widehat{d}(V, b c a)=N(S, \ldots, S, 0, \ldots, 0, S), \\
d_{4} & =\widehat{d}(V, b a c)=N(B, \ldots, B, T, \ldots, T, C) .
\end{aligned}
$$

Clearly, under STV candidate $a$ is the unique winner in $V$. Thus, it must be the case that $\min \left\{d_{1}, d_{2}\right\}<\min \left\{d_{3}, d_{4}\right\}$. By symmetry we have $d_{1}=d_{3}$, and hence $d_{2}<d_{4}$. Also, by symmetry we get $d_{4}=N(T, \ldots, T, C, B, \ldots, B)$. Hence, by monotonicity $C<B$.

Now, consider the profile $W$ obtained by replacing the last voter in $V$ by a voter whose preferences are $c b a$. We have

$$
\begin{aligned}
& d_{1}^{\prime}=\widehat{d}(W, a b c)=N(0, \ldots, 0, S, \ldots, S, C), \\
& d_{2}^{\prime}=\widehat{d}(W, a c b)=N(T, \ldots, T, C, \ldots, C, S), \\
& d_{3}^{\prime}=\widehat{d}(W, b c a)=N(S, \ldots, S, 0, \ldots, 0, B), \\
& d_{4}^{\prime}=\widehat{d}(W, b a c)=N(B, \ldots, B, T, \ldots, T, S) .
\end{aligned}
$$

The STV-winner of $W$ is $b$, so we have $\min \left\{d_{1}^{\prime}, d_{2}^{\prime}\right\}>\min \left\{d_{3}^{\prime}, d_{4}^{\prime}\right\}$. Furthermore, by symmetry, we have $d_{3}^{\prime}=N(0, \ldots, 0, S, \ldots, S, B)$. As $C<B$, by monotonicity we conclude that $d_{1}^{\prime} \leq d_{3}^{\prime}$. This implies that $d_{2}^{\prime}>d_{4}^{\prime}$. However, by symmetry we have $d_{4}^{\prime}=N(T, \ldots, T, B, \ldots, B, S)$, so by monotonicity $d_{2}^{\prime} \leq d_{4}^{\prime}$, a contradiction.

We can use similar ideas to show that STV is not distance-rationalizable with respect to $\mathcal{U}$ and a neutral anonymous monotonic votewise distance.

Theorem 15 For three candidates, STV (together with any intermediate tie-breaking rule) is not $(\mathcal{U}, \widehat{d})$-rationalizable with respect to any neutral anonymous monotonic votewise distance $\widehat{d}$.

Proof Suppose to the contrary that STV can be rationalized with respect to weak unanimity via a neutral anonymous monotonic votewise distance $\widehat{d}$, where $d$ is the underlying distance over the votes, and $N$ is the corresponding norm.

Let $V$ be as in (11) and $W$ is obtained from $V$ as in the proof of Theorem 14 . Let numbers $C, S, T$ be also the same as in that proof. Set $m_{s c}=\min \{S, C\}, m_{s b}=$ $\min \{S, B\}$. Let $U_{a}$ and $U_{b}$ denote the closest to $V$ weak unanimity consensus profiles with winners $a$ and $b$, respectively. Clearly, when constructing $U_{a}$ (respectively, $U_{b}$ ), we pick for each vote in $V$ the closest vote that ranks $a$ (respectively, $b$ ) first; these choices are made independently for each vote in $V$. Thus, we have $d(b c a, a b c)=S$, $d(b c a, a c b)=C$ and $d(c a b, a b c)=S, d(c a b, a c b)=B$, so

$$
\widehat{d}\left(V, U_{a}\right)=N\left(0, \ldots, 0, m_{s c}, \ldots, m_{s c}, m_{s b}\right) .
$$

Also $d(a b c, b a c)=B, d(a b c, b c a)=S$, and $d(c b a, a b c)=C, d(c b a, a c b)=S$, hence

$$
\widehat{d}\left(V, U_{b}\right)=N\left(m_{s b}, \ldots, m_{s b}, 0, \ldots, 0, m_{s c}\right) .
$$

As $a$ wins in $V$, we have $\widehat{d}\left(V, U_{a}\right)<\widehat{d}\left(V, U_{b}\right)$, and therefore by symmetry and monotonicity $m_{s c}<m_{s b}$. 
Now, let $U_{a}^{\prime}$ and $U_{b}^{\prime}$ denote its closest to $W$ weak unanimity consensus profiles with winners $a$ and $b$, respectively. We have

$$
\begin{aligned}
\widehat{d}\left(W, U_{a}^{\prime}\right) & =N\left(0, \ldots, 0, m_{s c}, \ldots, m_{s c}, m_{s c}\right), \\
\widehat{d}\left(W, U_{b}^{\prime}\right) & =N\left(m_{s b}, \ldots, m_{s b}, 0, \ldots, 0, m_{s b}\right) .
\end{aligned}
$$

As $b$ wins in $W$, we have $\widehat{d}\left(W, U_{a}^{\prime}\right)>\widehat{d}\left(W, U_{b}^{\prime}\right)$, and therefore $m_{s c}>m_{s b}$, a contradiction.

Note that for any $p=1, \ldots, \infty$, all $\ell_{p}$-votewise distance are anonymous and monotonic, so Theorems 14 and 15 mean that STV cannot be distance-rationalized with respect to either strong or weak unanimity via any neutral $\ell_{p}$-votewise distance.

Finally, we remark that STV is not distance-rationalizable with respect to $\mathcal{C}$ either since it is not Condorcet-consistent. Note that Meskanen and Nurmi (2008) show that STV can be distance-rationalized with respect to $\mathcal{U}$. Their distance is neutral, but not votewise. Further, it is not immediately clear if their distance is polynomial-time computable. Thus, of all rules we have considered, STV is distance-rationalizable in the weakest possible sense.

\subsection{Monotonicity and The Main Characterization Theorem}

Let us now consider another important property of voting rules, namely monotonicity. Characterizing precisely which votewise rules are monotone seems much more difficult than for homogeneous rules. We are able, however, to give two fairly natural conditions on distances over votes that ensure monotonicity of the voting rule when distances satisfying these two conditions are coupled with matching consensus classes.

In this section we will not discuss the Condorcet consensus. The reason for this is twofold. First, it is well-known that Dodgson rule is not monotone (see Brandt (2009) for a recent survey of Dodgson rule's deficiencies) and since Dodgson voting is $\left(\mathcal{C}, \widehat{d}_{\text {swap }}\right)$-rationalizable and $\widehat{d}_{\text {swap }}$ appears to be about the best behaved distance one can think of, finding either a sufficient or necessary condition for a distance $d$ to satisfy so that a voting rule is $(\mathcal{C}, \widehat{d})$-rationalized and monotone seems challenging. The second reason is that $\mathcal{C}$ is, in some sense, the least "local" of the consensus classes we consider. As a result, conditions regarding just the distance among votes may be very hard to translate to conditions regarding the whole profile (and monotonicity is a "global" condition in the sense that the whole profile is involved). Also for simplicity, we focus on $\ell_{1}$-votewise rules and $\ell_{\infty}$-votewise rules.

Let $C$ be a set of candidates and let $d$ be a distance over votes. How can we specify a condition on $d$ so that voting rules rationalized using this distance are monotone? Intuitively, the condition should ensure that if candidate $c$ is a winner and some voter ranks him higher than before, then the distance to the consensus where $c$ was the winner decreases more than the distance to a consensus with any other winner. The next definition tries to capture this intuition.

Definition 11 Let $C$ be a set of candidates and let $d$ be a distance between votes over $C$. We say that $d$ is relatively monotone if for every candidate $c \in C$, each two preference orders $y$ and $y^{\prime}$ such that $y^{\prime}$ is identical to $y$ except that $y^{\prime}$ ranks $c$ higher than $y$, and every two preference orders $x$ and $z$ such that $x$ ranks $c$ first and $z$ does not, it holds that

$$
d(x, y)-d\left(x, y^{\prime}\right) \geq d(z, y)-d\left(z, y^{\prime}\right) .
$$


As a quick sanity check, it is easy to observe that the swap distance, $d_{\text {swap }}$, satisfies the relative monotonicity condition.

Relative monotonicity of a distance $d$ over $P(C)$ naturally translates into the monotonicity of a $(\mathcal{K}, \widehat{d})$-rationalizable voting rule, provided that $\mathcal{K}$ is $\mathcal{S}$ or $\mathcal{U}$.

Theorem 16 Let $\mathcal{R}$ be a voting rule rationalized by $(\mathcal{K}, \widehat{d})$, where $\mathcal{K} \in\{\mathcal{S}, \mathcal{U}\}$ and $d$ is a relatively monotone distance on votes. Then $\mathcal{R}$ is monotone.

Proof Let $E=(C, V)$ be an election, where $V=\left(v_{1}, \ldots, v_{n}\right)$, and $c \in C$ be a candidate such that $c \in \mathcal{R}(E)$. Let $E^{\prime}=\left(C, V^{\prime}\right)$, where $V^{\prime}=\left(v_{1}^{\prime}, \ldots, v_{n}^{\prime}\right)$, be an arbitrary election that is identical to $E$ except one voter, say $v_{1}^{\prime}$, ranks $c$ higher ceteris paribus. It suffices to show that $c \in \mathcal{R}\left(E^{\prime}\right)$.

To show this, we give a proof by contradiction. Let $(C, U) \in \mathcal{K}$, where $U=$ $\left(u_{1}, \ldots, u_{n}\right)$, be a consensus witnessing that $c \in \mathcal{R}(E)$, and let $(C, W)$, where $W=$ $\left(w_{1}, \ldots, w_{n}\right)$, be any consensus in $\mathcal{K}$ such that $c$ is not a consensus winner of $(C, W)$. For the sake of contradiction, let us assume that $\widehat{d}\left(U, V^{\prime}\right)>\widehat{d}\left(W, V^{\prime}\right)$. If $\mathcal{K}$ is either $\mathcal{U}$ or $\mathcal{S}$, then we know that $u_{1}$ ranks $c$ first and that $w_{1}$ does not rank $c$ first. By relative monotonicity, this means that

$$
d\left(u_{1}, v_{1}\right)-d\left(u_{1}, v_{1}^{\prime}\right) \geq d\left(w_{1}, v_{1}\right)-d\left(w_{1}, v_{1}^{\prime}\right) .
$$

However, since $\widehat{d}\left(U, V^{\prime}\right)>\widehat{d}\left(W, V^{\prime}\right)$ and $V$ differs from $V^{\prime}$ only by the first voter, it holds that

$$
d\left(u_{1}, v_{1}^{\prime}\right)+\sum_{i=2}^{n} d\left(u_{i}, v_{i}\right)>d\left(w_{1}, v_{1}^{\prime}\right)+\sum_{i=2}^{n} d\left(w_{i}, v_{i}\right)
$$

If we add inequality (12) to inequality (13), we obtain

$$
d\left(u_{1}, v_{1}\right)+\sum_{i=2}^{n} d\left(u_{i}, v_{i}\right)>d\left(w_{1}, v_{1}\right)+\sum_{i=2}^{n} d\left(w_{i}, v_{i}\right)
$$

That is, $\widehat{d}(U, V)>\widehat{d}(W, V)$, which is a contradiction by our choice of $U$.

Relative monotonicity is a remarkably strong condition, not satisfied even by some very natural distances that, intuitively, should be monotone. Here is an example.

Example 1 Consider a scoring vector $\alpha=(0,1,2,3,4,5)$, i.e., the 6-candidate scoring vector for Borda rule and a candidate set $C=\left\{c, d, x_{1}, x_{2}, x_{3}, x_{4}\right\}$. Distance $d_{\alpha}$ does not satisfy the relative monotonicity condition. Indeed, let us consider the following four votes:

$$
\begin{gathered}
x: c>d>x_{1}>x_{2}>x_{3}>x_{4}, \\
z: x_{1}>c>x_{2}>x_{3}>x_{4}>d, \\
y: x_{1}>x_{2}>d>c>x_{3}>x_{4}, \\
y^{\prime}: x_{1}>x_{2}>c>d>x_{3}>x_{4} .
\end{gathered}
$$

Note that $y$ and $y^{\prime}$ are identical except that in $y^{\prime}$ candidates $c$ and $d$ are swapped, $c$ is ranked on the top of $x$ and is not ranked on the top of $z$. We can easily verify that $d(x, y)-d\left(x, y^{\prime}\right)=0$ but $d(z, y)-d\left(z, y^{\prime}\right)=2$. Thus, $d_{\alpha}$ is not relatively monotone.

For the case of $\mathcal{U}$ we can weaken the assumptions of Theorem 16 to relative minmonotonicity defined below. It is easy to modify the proof. 
Definition 12 Let $C$ be a set of candidates and let $d$ be a distance between votes over $C$. We say that $d$ is relatively min-monotone if for each candidate $c \in C$ and each two preference orders $y$ and $y^{\prime}$ such that $y^{\prime}$ is identical to $y$ except that $y^{\prime}$ ranks $c$ higher than $y$, it holds that for each candidate $e \in C \backslash\{c\}$ :

$$
\min _{x \in \mathcal{P}(C, c)} d(x, y)-\min _{x^{\prime} \in \mathcal{P}(C, c)} d\left(x^{\prime}, y^{\prime}\right) \geq \min _{z \in \mathcal{P}(C, e)} d(z, y)-\min _{z^{\prime} \in \mathcal{P}(C, e)} d\left(z^{\prime}, y^{\prime}\right) .
$$

Corollary 2 Let $\mathcal{R}$ be a $(\mathcal{U}, \widehat{d})$-rationalized voting rule, where $d$ is relatively minmonotone distance over votes. Then $\mathcal{R}$ is monotone.

On the other hand, if we want to obtain monotonicity for voting rules based on votewise distances and the majority consensus, it seems that we have to give up the "relative" part of the definition of relative min-monotone distances. The next definition explains this formally.

Definition 13 Let $C$ be a set of candidates and let $d$ be a distance over votes over $C$. We say that $d$ is min-monotone if for every candidate $c \in C$ and every two preference orders $y$ and $y^{\prime}$ such that $y^{\prime}$ is the same as $y$ except that it ranks $c$ higher, for each $e \in C \backslash\{c\}$ it holds that:

$$
\begin{aligned}
& \min _{x \in \mathcal{P}(C, c)} d(x, y) \geq \min _{x^{\prime} \in \mathcal{P}(C, c)} d\left(x^{\prime}, y^{\prime}\right), \\
& \min _{z \in \mathcal{P}(C, e)} d(z, y) \leq \min _{z^{\prime} \in \mathcal{P}(C, e)} d\left(z^{\prime}, y^{\prime}\right) .
\end{aligned}
$$

In other words, a distance is min-monotone if ranking a certain candidate $c$ higher never increases his distance from the closest vote where he is ranked first, and, for every candidate $e$ other than $c$, never decreases his distance from a vote where $e$ is ranked first. It is easy to see that relative min-monotonicity is a relaxation of minmonotonicity and so Corollary 2 applies to min-monotone distances as well. Let us state this for further references:

Proposition 8 Each min-monotone distance d over votes is relatively min-monotone.

Using min-monotone distances, we can now show an analog of Theorem 16 for the case of the majority consensus.

Theorem 17 Let $\mathcal{R}$ be a $(\mathcal{M}, \widehat{d})$-rationalizable voting rule where $d$ is a min-monotone distance on votes. Then $\mathcal{R}$ is monotone.

Proof Let $E=(C, V)$ be an election with $V=\left(v_{1}, \ldots, v_{n}\right)$ and let $c \in \mathcal{R}(E)$ be one of the winners of $E$. Let $(C, U)$, where $U=\left(u_{1}, \ldots, u_{n}\right)$, be a majority consensus closest to $V$ with $c$ being its majority winner. Let $E^{\prime}=\left(C, V^{\prime}\right)$, where $V^{\prime}=\left(v_{1}^{\prime}, v_{2}, \ldots, v_{n}\right)$ which differ from $V$ only in the first preference order and $v_{1}^{\prime}$ is identical to $v_{1}$ except that it ranks $c$ higher than $v_{1}$.

For the sake of contradiction, we assume that $c$ is not an $\mathcal{R}$ winner of $E^{\prime}$, but that some candidate $e \in C \backslash\{c\}$ is. Let $(C, W)$, where $W=\left(w_{1}, \ldots, w_{n}\right)$ be a majority consensus closest to $V^{\prime}$ with $e$ being a simple majority winner of $E^{\prime}$. Let us form two new $\mathcal{M}$-consensuses, $U^{\prime}$ and $W^{\prime}$ as follows:

1. $U^{\prime}=\left(u_{1}^{\prime}, u_{2}, \ldots, u_{n}\right)$, where $u_{1}^{\prime}=v_{1}^{\prime}$, if $u_{1}$ does not ranks $c$ first and $u_{1}^{\prime} \in$ $\arg \min _{x \in \mathcal{P}(C, c)} d\left(x, v_{1}^{\prime}\right)$ otherwise. The majority winner of this new consensus is again $c$. 
2. $W^{\prime}=\left(w_{1}^{\prime}, w_{2}, \ldots, w_{n}\right)$, where $w_{1}^{\prime}=w_{1}$, if $w_{1}$ does not ranks $e$ first and $w_{1}^{\prime} \in$ $\arg \min _{z \in \mathcal{P}(C, c)} d\left(z, w_{1}^{\prime}\right)$ otherwise. The majority winner of this new consensus is again $e$.

Thus, by Lemma 1 and min-monotonicity of $d$

$$
d\left(u_{1}^{\prime}, v_{1}^{\prime}\right) \leq d\left(u_{1}, v_{1}\right), \quad d\left(w_{1}^{\prime}, v_{1}^{\prime}\right) \geq d\left(w_{1}, v_{1}\right) .
$$

Now, using the fact that $V$ and $V^{\prime}$ agree on all voters but the first one, our choice of $W^{\prime}$, and the two above inequalities, we can see that the following inequality holds:

$$
\begin{aligned}
\widehat{d}(U, V) & =d\left(u_{1}, v_{1}\right)+\sum_{i=2}^{n} d\left(u_{i}, v_{i}\right) \geq d\left(u_{1}^{\prime}, v_{1}^{\prime}\right)+\sum_{i=2}^{n} d\left(u_{i}, v_{i}\right) \\
& >d\left(w_{1}^{\prime}, v_{1}^{\prime}\right)+\sum_{i=2}^{n} d\left(w_{i}, v_{i}\right) \geq d\left(w_{1}, v_{1}\right)+\sum_{i=2}^{n} d\left(w_{i}, v_{i}\right)=\widehat{d}(W, V) .
\end{aligned}
$$

However, this is a contradiction because by our choice of $U, \widehat{d}(U, V)$ is a minimal distance between $V$ and any majority consensus with $n$ voters.

We can use essentially the same proof for the case of $\ell_{\infty}$-votewise distances.

Corollary 3 Let $\mathcal{R}$ be a $\left(\mathcal{M}, \widehat{d^{\infty}}\right)$-rationalizable voting rule, where $d$ is a min-monotone distance on votes. Then $\mathcal{R}$ is monotone.

Note that it is hard to apply the notion of min-monotone distances to prove monotonicity of voting rules that are distance rationalized via strong unanimity consensus.

The next proposition, together with Example 1, shows that indeed min-monotonicity is a considerably weaker condition than relatvie monotonicity.

Proposition 9 Let $\alpha=\left(\alpha_{1}, \ldots, \alpha_{m}\right)$ be a nonnegative scoring vector. Pseudodistance $d_{\alpha}$ is min-monotone if and only if $\alpha$ is nonincreasing.

Proof As it follows from its definition given in (4) the vector $\alpha$ and vector

$$
\beta=\left(\alpha_{1}-a, \alpha_{2}-a, \ldots, \alpha_{m}-a\right)
$$

for any $a$ generate the same distance. It will be convenient to choose $a=\alpha_{1}$ and getting $\beta_{1}=0$ (allowing apparently by doing so negative weights). As we showed in the proof of Theorem 1 for each candidate $c \in C$, each integer $k$ such that $1 \leq k \leq m$, and each vote $y$ that ranks $c$ in position $k$, we have $\min _{x \in \mathcal{P}(C, c)} d(x, y)=2\left|\beta_{1}-\beta_{k}\right|=2\left|\beta_{k}\right|$. Let us fix some two distinct candidates $c, e \in C$. Let $y$ and $y^{\prime}$ be two votes that are identical, except that $c$ is ranked on some position $k$ in $y$ and in $y^{\prime}$ candidate $c$ is shifted to position $\ell$, where $\ell<k$. We know that $\min _{x \in \mathcal{P}(C, c)} d(x, y)=2\left|\beta_{1}-\beta_{k}\right|=2\left|\beta_{k}\right|$ and $\min _{x^{\prime} \in \mathcal{P}(C, c)} d\left(x^{\prime}, y^{\prime}\right)=2\left|\beta_{1}-\beta_{\ell}\right|=2\left|\beta_{\ell}\right|$. If $\alpha$ is nonincreasing, all $\beta_{i}$ are nonpositive then (14) is satisfied if and only if $\left|\beta_{\ell}\right| \leq\left|\beta_{k}\right|$ which is in turn equivalent to $\beta_{\ell} \geq \beta_{k}$ and $\alpha_{\ell} \geq \alpha_{k}$.

Thus, the first inequality (14) from the definition of min-monotonicity is satisfied if and only if $\alpha$ is nondecreasing. One can analogously show that the same holds for the second inequality (in essence, the proof works by arguing that either $\operatorname{pos}_{y}(e)=\operatorname{pos}_{y^{\prime}}(e)$ or $\operatorname{pos}_{y}(e)=\operatorname{pos}_{y^{\prime}}(e)-1$. 
The above proposition, combined with Theorem 7 give us our main characterization theorem

Theorem 18 A voting rule $\mathcal{R}$ is $(U, \widehat{d})$-rationalizable for some min-monotone neutral pseudodistance $d$ on votes if and only if $\mathcal{R}$ is a scoring rule $\mathcal{R}_{\alpha}$ for some vector $\alpha=$ $\left(\alpha_{1}, \ldots, \alpha_{m}\right)$ with $\alpha_{1} \geq \alpha_{2} \geq \ldots \geq \alpha_{m}$.

\section{Complexity Issues for Distance-Rationalizable Rules}

Now that we have some understanding of the nature of distance-rationalizable rules in general and votewise rules specifically, it is high time to consider their computational aspects. We will focus on two issues. Foremost, we will show complexitytheoretic upper-bounds on the runtime of winner-determination procedures for a large class of distance-rationalizable rules. Then, we will show that for votewise rules we can obtain stronger fixed-parameter tractability resutls. Finally, we will show that in a certain technical sense $\ell_{\infty}$-votewise rules can be considered as approximations to the corresponding $\ell_{1}$-votewise rules. This result is interesting because sometimes $\ell_{\infty}$ votewise rules have considerably more efficient winner-determination procedures than their $\ell_{1}$-votewise counterparts. (We will indeed see this for the case of Dodgson and its $\ell_{\infty}$-votewise analogue.)

We assume that the reader is familiar with basic notions of computational complexity theory such as complexity classes $\mathrm{P}$ and NP, (nondeterministic) polynomialtime computation, etc. In addition, our analysis will include complexity classes $\mathrm{P}^{\mathrm{NP}}$ and $\Theta_{2}^{p}$ that lie one notch higher in the Polynomial Hierarchy, and the class FPT of fixed-parameter tractable problems. A decision problem belongs to $\mathrm{P}^{\mathrm{NP}}$ if, given access to an NP-oracle, it can be solved in polynomial time (i.e., the problem can be solved in polynomial-time given a procedure that solves some given NP-problem in constant time). A decision problem is in $\Theta_{2}^{p}$ if it is in $\mathrm{P}^{\mathrm{NP}}$ but all the oracle queries are prepared before any of the answers are received (in other words, the queries cannot be chosen adaptively). A catalog of reductions and complexity classes can be found in (L. Hemaspaandra \& Ogihara, 2002). A problem is fixed-parameter tractable (is in FPT) with respect to some parameter (in our case the parameter will be the number of candidates in the election) if there is an algorithm that for each instance $I$ of size $n$ with parameter value $j$ computes the solution to the problem in time $O\left(f(j) n^{O(1)}\right)$, where $f$ is a (computable) function of $j$.

\subsection{Winner Determination Problem}

For a given voting rule $\mathcal{R}$ to be of any practical value, it must be possible to efficiently establish $\mathcal{R}$-winners of elections. Let us consider the complexity of winnerdetermination under distance-rationalizable rules. Our first goal is to prove upper bounds on the hardness of winner determination. However, to do so we, of course, need to impose restrictions on the distances that we consider. Otherwise, due to our unrestricted distance-rationalizability results no upper bound would exist. Thus, we will focus on distances that are polynomial-time computable and take values from the set $\mathbb{Z} \cup\{\infty\}$. 
Definition 14 Let $d$ be a distance over some set $X$. We say that $d$ is normal if it is polynomial-time computable (given an encoding of the elements in $X$ ) and takes values from the set $\mathbb{Z} \cup\{\infty\}$.

Naturally, for us the set $X$ will either be the set of all preference orders over some candidate set or the set of all profiles over some candidate set. We require a distance to have values in $\mathbb{Z} \cup\{\infty\}$ for technical reasons only; standard mechanism of computational complexity can easily deal with integers (and could be adopted to work with rationals) but have no clear way of dealing with irrational numbers. Nonetheless, essentially all natural, interesting distances are normal.

Formally, we define this winner-determination problem as follows.

Definition 15 Let $\mathcal{R}$ be a voting rule. In the $\mathcal{R}$-winner problem we are given an election $E=(C, V)$ and a candidate $c \in C$, and we ask whether $c \in \mathcal{R}(E)$.

Our next theorem provides complexity-theoretic upper bounds for $\mathcal{R}$-winner determination where $\mathcal{R}$ is distance-rationalizable via a normal distance $D$ and one of our standard consensus classes.

Theorem 19 Suppose that a voting rule $\mathcal{R}$ is $(\mathcal{K}, D)$-rationalizable, where $D$ is a normal distance that for any two elections $E_{1}=\left(C, V_{1}\right), E_{2}=\left(C, V_{2}\right)$ satisfies $D\left(E_{1}, E_{2}\right)=+\infty$ whenever $C_{1} \neq C_{2}$ or $\left|V_{1}\right| \neq\left|V_{2}\right|$, and $\mathcal{K} \in\{\mathcal{S}, \mathcal{U}, \mathcal{M}, \mathcal{C}\}$. It holds that:

1. $\mathcal{R}$-winner is in $\mathrm{P}^{\mathrm{NP}}$, and

2. if, in addition, there is a polynomial $p$ such that for each two elections $E_{1}$ and $E_{2}$ either $D\left(E_{1}, E_{2}\right)=+\infty$ or $D\left(E_{1}, E_{2}\right) \leq p\left(|C|+\left|V_{1}\right|+\left|V_{2}\right|\right)$ holds, then $\mathcal{R}$-winner is in $\Theta_{2}^{p}$.

Proof Let $E=(C, V)$ be our input election and let $c$ be a candidate in $C$. Our goal is to decide whether $c \in \mathcal{R}(E)$. In our construction we will use the following decision problem, which we call $\mathcal{R}$-score. Given an election $F$, a candidate $e$ in this election, and a nonnegative integer $k$ we ask if $(\mathcal{K}, D)$-score of $e$ is at most $k$. It is easy to see that since $D$ is normal (and, as a result, polynomial-time computable), $\mathcal{R}$-score is in NP.

To show that $\mathcal{R}$-winner is in $\mathrm{P}^{\mathrm{NP}}$ we can use the following simple algorithm: (a) For each candidate $e \in C$, using binary-search and oracle queries to $\mathcal{R}$-score, compute the $(\mathcal{K}, D)$-score of $e$. If $c$ is among the candidates with the smallest $(\mathcal{K}, D)$-score then accept and otherwise reject. Since $D$ is polynomial-time computable, we know that $(\mathcal{K}, D)$-score of each candidate in $E$ is exponentially bounded in $|C|+|V|$ (or, is $+\infty$ ); this is so because whatever polynomial-time algorithm we would use for computing the value of $D$, would not have time to output more than polynomially many bits. Thus, the binary search procedure would require at most polynomially many queries per candidate and the whole procedure would run in polynomial time (given access to $\mathcal{R}$-score oracle).

Let us now consider the second statement of the theorem. To establish membership in $\Theta_{2}^{p}$, we will provide an algorithm that solves $\mathcal{R}$-winner in polynomial-time, provided it is allowed to make independent oracle queries to $\mathcal{R}$-score (i.e., the algorithm will form all the queries prior to receiving any of the oracle answers). Clearly, this means that the algorithm cannot use binary search directly. Nonetheless, in the second statement of the theorem we have assumed that the values of $D$ are either $+\infty$ or are polynomially 
bounded with respect to $|C|+|V|$ (recall that if $D\left(E_{1}, E_{2}\right) \neq+\infty$, then $\left|V_{1}\right|=\left|V_{2}\right|$ ). As a result, each candidate in $C$ has $(\mathcal{K}, D)$-score that is $+\infty$ or that is polynomially bounded with respect to $|C|+|V|$.

Our algorithm works as follows. For each candidate $e \in C$ and for each integer $k$ that $e$ could possibly have as the score, our algorithm queries the oracle whether $e$ has $(\mathcal{K}, D)$-score at most $k$. After all these queries are asked, we can trivially compute each candidate's score and output the winner. Clearly, we make at most polynomially many queries and the whole algorithm runs in polynomial time.

It seems that Theorem 19 cannot be improved. Indeed, it is well-known that winner determination under Dodgson's and Kemeny's rules, both of which satisfy the second part of the theorem, is $\Theta_{2}^{p}$-complete (E. Hemaspaandra et al., 1997; E. Hemaspaandra, Spakowski, \& Vogel, 2005). For the first part of the theorem, it is not too difficult to construct a distance which is not polynomially bounded; e.g., consider a family $\mathcal{R}$ of scoring rules $\mathcal{R}_{\alpha_{m}}, m=1,2, \ldots$, where the coefficients of $\alpha_{m}$ depend exponentially on $m$. While for such scoring rules the winner determination procedure is much easier than $\mathrm{P}^{\mathrm{NP}}$-complete (even if the rule is represented by a distance and not the score vector itself), it seems that the distances used to rationalize such scoring rules would yield rules with $\mathrm{P}^{\mathrm{NP}}$-complete winner determination problems when paired with either $\mathcal{C}$ or $\mathcal{S}$. However, no such result was yet proved since such rules do not seem practically interesting.

On the positive side, for both Dodgson and Kemeny the winner determination problem can be solved in polynomial time if the number of candidates is fixed. In fact, a stronger statement is true: The winner determination problem for both Dodgson and Kemeny is fixed parameter tractable with respect to the number of candidates. This is an example of a more general fact: If a voting rule is rationalized via a normal $\ell_{1}$ votewise distance, the winner determination problem is fixed-parameter tractable with respect to the number of candidates.

Theorem 20 Suppose that a voting rule $\mathcal{R}$ is $(\mathcal{K}, \widehat{d})$-rationalizable, where $\mathcal{K} \in\{\mathcal{S}, \mathcal{U}, \mathcal{M}, \mathcal{C}\}$. and $\widehat{d}$ is a normal $\ell_{1}$-votewise distance. Then the $\mathcal{R}$-winner problem is in FPT with respect to the number of candidates.

Proof There is an FPT algorithm, due to Lenstra, Jr. (1983), that - in effect - allows us to solve integer linear programs for which the number of variables is a function of the parameter (and for which the constants in our integer linear programs are wellbehaved; in our case they will be). Thus, our proof proceeds by showing an integer linear program for computing $(\mathcal{K}, \widehat{d})$-score of a given candidate, whose number of variables will be a function of the number of candidates in the election. Naturally, the ability to compute $(\mathcal{K}, \widehat{d})$-scores immediately implies the ability to compute winners.

Let $E=(C, V)$ be an election and let $e$ be a candidate in $C$. Let $\rho_{1}, \ldots, \rho_{|C| !}$ be all $|C|$ ! preference orders over $C$. Our integer linear program for $e$ 's $(\mathcal{K}, \widehat{d})$-score at a profile $V=\left(v_{1}, \ldots, v_{n}\right)$ is as follows. Given another profile $U=\left(u_{1}, \ldots, u_{n}\right)$ we can, for every $v, u \in P(C)$ calculate the number $n_{v, u}$ equal to the number of indices $i \in\{1,2, \ldots, n\}$ such that $v=v_{i}$ and $u=u_{i}$. The set of numbers $N_{V, U}=\left\{n_{v, u} \mid v, u \in P(C)\right\}$ does not determine $U$ uniquely but any two profiles $U_{1}$ and $U_{2}$ with $N_{V, U_{1}}=N_{V, U_{2}}$ have the property $\widehat{d}\left(V, U_{1}\right)=\widehat{d}\left(V, U_{2}\right)$. 
In the program we seek to minimize the distance from $V$ to a consensus profile $U$, represented by $N_{V, U}$, where $e$ is the winner. That is, we seek to minimize

$$
\sum_{i=1}^{|C| !|C| !} \sum_{j=1} n_{\rho_{i}, \rho_{j}} d\left(\rho_{i}, \rho_{j}\right)
$$

(which, will then be $d$ 's $(\mathcal{K}, \widehat{d})$-score) under the following constraints:

1. For each $i, 1 \leq i \leq|C|$ !, the sum $\sum_{j=1}^{|C| !} n_{\rho_{i}, \rho_{j}}$ equals the number of voters in $V$ with preference order $\rho_{i}$ (that is, the variables really correspond to the voters in $V$ ).

2. If we form an election where for each $j, 1 \leq j \leq|C|$ !, there are exactly $\sum_{i=1}^{|C| !} n_{\rho_{i}, \rho_{j}}$ voters with preference $\rho_{j}$, then this election is a $\mathcal{K}$-consensus with $e$ as the consensus winner.

The first constraint above is clearly in the form of a linear equation, as required by integer linear programs. The second constraint can also be expressed in terms of linear inequalities for each of the consensus classes we consider. This completes the proof.

The above two theorems give complexity-theoretic upper bounds for hardness of winner determination under distance-rationalizable/votewise rules. While there are some settings where these upper bounds are exact (consider, e.g., Dodgson and Kemeny), it seems to not be the case for $\ell_{1}$-votewise rules rationalized via the unanimity consensus. Indeed, in prior sections we have seen that such rules form families of scoring rules, and for scoring rules winner determination is next to trivial. Note, however, that in our setting we are given the distance, but not the scoring vector and computing the latter from the former might be hard. Nevertheless, it turns out that in this setting (and in similar ones) we can easily determine the winner if we are allowed to use polynomial-size advice.

Theorem 21 Suppose that a voting rule $\mathcal{R}$ is distance-rationalizable via a normal neutral $\ell_{1}$-votewise ( $\ell_{\infty}$-votewise) distance and either the unanimity consensus or the majority consensus. Then $\mathcal{R}$-winner is in $\mathrm{P} /$ poly.

$\mathrm{P} /$ poly is a complexity class that captures the power of polynomial computation "with advice." (Informally, here the advice is the scoring vector underlying the rule.) KarpLipton theorem (Karp \& Lipton, 1980) says that if there is an NP-hard problem in $\mathrm{P} /$ poly then the Polynomial Hierarchy collapses. Thus, for voting rules that are distance-rationalizable via a normal neutral $\ell_{1}$-votewise distance and the consensus class $\mathcal{U}$ the winner determination problem is unlikely to be NP-hard. This stands in sharp contrast with such rules rationalized via consensus classes $\mathcal{C}$ and $\mathcal{S}$, which indeed can have NP-hard winner determination problems (again, Dodgson and Kemeny server as examples). Thus, from computational perspective, the unanimity consensus appears to be easier to work with than the strong unanimity consensus and the Condorcet consensus. Indeed, both $\mathcal{S}$ and $\mathcal{C}$ impose "global" constraints on the closest consensus and $\mathcal{U}$ only imposes "local" ones.

\section{$5.2 \ell_{1}$-Votewise Rules versus $\ell_{\infty}$-Votewise Rules}

In addition to complexity-theoretic analysis of winner determination, it is also interesting to consider relations between winner determination in rules defined via similar 
distances and consensus classes. Specifically, we will now discuss the relation between $\ell_{1}$-votewise rules and $\ell_{\infty}$-votewise rules. It turns out that in a certain weak sense, $\ell_{\infty}$ votewise rules are approximations of corresponding $\ell_{1}$-votewise rules using the same distance over votes and the same consensus class.

Theorem 22 Let $\mathcal{K} \in\{\mathcal{S}, \mathcal{U}, \mathcal{M}, \mathcal{C}\}$ be a consensus class and let d be a distance over votes. Let $\widehat{d}$ be an $\ell_{1}$ extension of $d$ to a distance over elections and $\widehat{d}^{\infty}$ be an $\ell_{\infty}$ extension of $d$. Let $\mathcal{R}$ be $(\mathcal{K}, \widehat{d})$-rationalized and $\mathcal{R}^{\infty}$ be $\left(\mathcal{K}, \widehat{d}^{\infty}\right)$-rationalized. For each election $E=(C, V)$ and each candidate $c \in C$ it holds that

$$
\operatorname{score}_{\mathcal{R} \infty}(c) \leq \operatorname{score}_{\mathcal{R}}(c) \leq|V| \cdot \operatorname{score}_{\mathcal{R}} \infty(c) .
$$

Proof Let $E=(C, V)$ be an arbitrary election, where $C=\left\{c_{1}, \ldots, c_{m}\right\}$ and $V=$ $\left(v_{1}, \ldots, v_{n}\right)$. We fix candidate $c \in C$.

We first claim that $\operatorname{score}_{\mathcal{R}}(c) \leq|V| \cdot \operatorname{score}_{\mathcal{R}^{\infty}}(c)$. Let $(C, W)$, where $W=\left(w_{1}, \ldots, w_{n}\right)$, be a $\mathcal{K}$-consensus where $c$ is the winner and such that score $_{\mathcal{R}^{\infty}}(c)=\widehat{d}^{\infty}(V, W)$. By definition, we have

$$
\begin{aligned}
\operatorname{score}_{\mathcal{R}}(c) \leq \widehat{d}(V, W) & =\sum_{i=1}^{n} d\left(v_{i}, w_{i}\right) \leq n \cdot \max _{i=1}^{n}\left\{d\left(v_{i}, w_{i}\right)\right\} \\
& =n \cdot \widehat{d}^{\infty}(V, W)=|V| \cdot \operatorname{score}_{\mathcal{R}} \infty(c)
\end{aligned}
$$

which proves the second inequality.

On the other hand, let $(C, U)$, where $U=\left(u_{1}, \ldots, u_{n}\right)$, be a $\mathcal{K}$-consensus where $c$ is the winner and for which score $\mathcal{R}_{\mathcal{R}}(c)=\widehat{d}(V, U)$. By definition, it holds that

$$
\begin{aligned}
\operatorname{score}_{\mathcal{R}}(c)=\widehat{d}(V, U) & =\sum_{i=1}^{n} d\left(v_{i}, u_{i}\right) \geq \max _{i=1}^{n}\left\{d\left(v_{i}, u_{i}\right)\right\} \\
& =\widehat{d}^{\infty}(V, U) \geq \operatorname{score}_{\mathcal{R}} \infty(c),
\end{aligned}
$$

and so score $_{\mathcal{R}} \infty(c) \leq \operatorname{score}_{\mathcal{R}}(c)$. This completes the proof.

In other words, an $\ell_{\infty}$-votewise rule is a $|V|$-approximation of a corresponding $\ell_{1}$ votewise rule in the sense of Caragiannis et al. (Caragiannis et al., 2009; Caragiannis, Kaklamanis, Karanikolas, \& Procaccia, 2010). Of course, this approximation is very weak as its approximation ratio depends linearly on the number of voters. The only settings where such an approximation would be of any value would be those, where we have very few voters and very many candidates (and the original voting rule is hard to compute). One of the few settings where these conditions are satisfied is the situation where we view web search engines as voting on the most appropriate result for a given query. There are very few search engines, but millions of web pages. (See the classic paper of Dwork et al. (Dwork, Kumar, Naor, \& Sivakumar, 2001) for a discussion of the idea of search-engine elections.) If we were for some reason to use an approximation algorithm for Dodgson's rule, then the algorithms of Caragiannis et al. (Caragiannis et al., 2009, 2010), whose approximation ratios depend on functions of $|C|$, would eventually give higher approximation ratios than $|V|$, yielded by the $\left(\mathcal{C}, d_{\text {swap }}^{\infty}\right)$-rationalizable rule $\mathcal{R}$. Of course, for $\mathcal{R}$ to be useful, we would have to be able to compute its winners in polynomial time. This is indeed the case. 
Proposition 10 Let $\mathcal{R}$ be $\left(\mathcal{C}, d_{\mathrm{swap}}^{\infty}\right)$-rationalizable. The problem of deciding whether a given candidate is a winner of $\mathcal{R}$-elections is in $\mathrm{P}$.

Proof Let $E=(C, V)$ be an election and let $c \in C$ be an arbitrary candidate. It is easy to verify that the following algorithm computes $\mathcal{R}$-score of $c$ in $E$ :

1. Set $s=0$.

2. If $c$ is a Condorcet winner of $E$ then return $s$.

3. For each vote where $c$ is not ranked first, swap $c$ and its preceding candidate.

4. Increase $s$ by 1 .

5. Go to Step 2.

Since after at most $|V|$ steps the algorithm terminates, it is easy to see that it runs in polynomial time. This completes the proof.

It is not at all clear that it is always the case, or at least the case for nice distances, that an $\ell_{\infty}$ variant of an $\ell_{1}$-votewise rule has a polynomial-time winner-determination procedure.

Question 1 Let $R$ be $\left(\mathcal{S}, d_{\text {swap }}^{\infty}\right)$-rationalizable. Is the problem of deciding whether a given candidate is a winner of $\mathcal{R}$-elections in $\mathrm{P}$ ?

Note that the rule in the above question is an $\ell_{\infty}$ variant of Kemeny. Of course, there are much better approximation algorithms known for Kemeny (Ailon, Charikar, \& Newman, 2008; Coppersmith, Fleisher, \& Rurda, 2010; Kenyon-Mathieu \& Schudy, 2007) and the value of resolving the above question is in enhancing our understanding of Kemeny and relations between $\ell_{1}$ - and $\ell_{\infty}$-votewise rules.

\section{Distance Rationalizable Rules and Rules that are Maximum Likelihood Estimators}

Condorcet viewed voting as aggregation of information. He assumed that there always exists a "correct" outcome (winner or ranking), and each voter's vote corresponds to a noisy perception of this correct outcome. This line of research was actively pursued by Young (1975) and Young and Levenglick (1978) who showed that consistent application of Condorcet's principles leads to the unique rule which is known as Kemeny rule. Recently the idea was further explored by Conitzer with coauthors (Conitzer \& Sandholm, 2005; Conitzer et al., 2009).

Maximum likelihood estimator (MLE) voting rules and votewise distance rationalisable (VDR) voting rules have some rules in common. This section is devoted to establishing connections between these two classes.

Firstly, we note that two possible assumptions: existence of a correct ranking and existence of a correct winner lead to two slightly different frameworks MLERIV and MLEWIV, respectively, in terminology of Conitzer \& Sandholm (2005). However in both cases the central concept of an MLE voting rule is the model of noise. Let us deal with the ranking case first.

Definition 16 A noise model (in the ranking case) is a family of probability distributions $P(x \mid y)$ on $P(C)$ indexed by $y \in P(C)$. This is the probability that a voter would submit preference order $x$ if the correct ranking was $y$. 
We assume that each voter is influenced with the same factors that cause her to make mistakes in her judgement. We also assume that the probability distribution $P(x \mid y)$ is the same for all voters, that is, the voters' preference orders are independent identically distributed (iid) random variables. In the classical framework of the jury deciding on whether or not a defendant is guilty or not we may have for example for each juror probabilities:

$$
P(g \mid g)=0.9, \quad P(i \mid g)=0.1, \quad P(g \mid i)=0.2, \quad P(i \mid i)=0.8,
$$

where 'g' stands for 'guilty' verdict preferred to 'innocent' verdict and 'i' stands for the opposite preference.

Definition 17 A social welfare function $F: P(C)^{n} \rightarrow P(C)$ is a maximum likelihood estimator (MLE) if there exists a noise model $P$ such that

$$
F(V)=\operatorname{argmax}_{x \in P(C)} \prod_{i=1}^{n} P\left(v_{i} \mid x\right)
$$

for any positive integer $n$ and any profile $V=\left(v_{1}, \ldots, v_{n}\right) \in P(C)^{n}$.

It is inevitable that we have to have a separate noise model for each number of alternatives $m$. But it would be unreasonable to allow the noise to depend on the number of voters $n$ (as we mentioned all voters are in the same conditions and if more voters join, this does not change those conditions). Hence it is important to emphasise that since we define a rule applicable for a fixed number of alternatives $m$ but for an arbitrary number of voters $n$ in the above definition the noise does not depend on $n$.

Voting rules that calculate the MLE social welfare function first and then harvest the top alternative(s) are MLERIV (maximum likelihood estimator for ranking under identically distributed independent votes) rules (Conitzer \& Sandholm, 2005).

Definition 18 A noise model is said to be neutral if $P(\tilde{\pi}(x) \mid \tilde{\pi}(y))=P(x \mid y)$ for any $x, y \in P(C)^{n}$ and any permutation $\pi \in S_{m} .^{7}$

The noise model (16) is obviously not neutral because the probabilities conditional on $g$ are different from the probabilities conditional on $i$ and this could not happen with a neutral noise model.

One of the most popular noise models in the literature is the Mallows model (Mallows, 1957). His idea was to use the Kendall tau distance to introduce a probability measure on $S_{m}$ (which can be identified with $P(C)$ for our needs). This measure has a location parameter $x_{0} \in P(C)$ and a scale parameter $\lambda$ which is a non-negaitive real number. Following Diaconis (1988) we generalise this measure by using an arbitrary pseudodistance $^{8} d$ on $P(C)$ by setting

$$
P\left(x \mid x_{0}\right)=c e^{-\lambda d\left(x, x_{0}\right)}, \quad c=\left(\sum_{x \in P(C)} e^{-\lambda d\left(x, x_{0}\right)}\right)^{-1} .
$$

We will call it the generalised Mallows model of noise relative to the pseudodistance $d$.

7 The notation $\tilde{\pi}$ was introduced in Section 4.1.

8 As Diaconis (1988) (p. 102) does not require the metric to satisfy the identity of indiscernibles. 
Here the largest probability is assigned to $x_{0}$ and probability decreases geometrically as a function of the distance from $x_{0}$. Increasing $\lambda$ makes the distribution more and more peaked about $x_{0}$. Critchlow (1985) provides a number of examples where the Mallows model provides a good fit to ranking data.

Theorem 23 A rule $\mathcal{R}$ is $(\mathcal{S}, \widehat{d})$-rationalizable relative to the strong consensus class $\mathcal{S}$ and additively votewise distance $\widehat{d}$ if and only if $\mathcal{R}$ is a MLERIV rule relative to the generalised Mallows model of noise.

Proof Let $P(V \mid x)$ be the probability of profile $V$, given the correct ranking $x$. We have

$$
P(V \mid x)=\prod_{i=1}^{n} P\left(v_{i} \mid x\right)=\prod_{i=1}^{n} c e^{-\lambda d\left(v_{i}, x\right)}=c e^{-\lambda \sum_{i=1}^{n} d\left(v_{i}, x\right)}=c e^{-\lambda \widehat{d}(V, X)},
$$

where $X$ is a strong consensus profile where all linear orders are equal to $x$. Hence maximising this probability is equivalent to minimising $\widehat{d}(V, X)$ and this is exactly what $\mathcal{R}$ is doing.

Since this is an if and only if statement we can obtain consequences in both directions. In particular we get a new MLERIV rule.

Corollary 4 Litvak's rule is MLERIV.

Among MLERIV rules Conitzer and Sandholm (2005) list scoring rules, Kemeny rule and STV. The presence of STV in this list seem to contradict to our Theorems 23 since we know that STV is not votewise distance rationalizable with respect to $\mathcal{S}$. Why is this happenning? There could be two answers to this question (but we do not know which one is true). Either STV requires the noise model different from the Mallows model or else it is impossible to come up with the noise model for STV which is independent of $n$ (it is dependent in Conitzer and Sandholm (2005)).

Now let us deal with the MLE rules which estimate the winner with no prior estimation of the ranking.

Definition 19 A noise model (in the winner case) is a family of probability distributions $P(k \mid a)$ on $A$ indexed by $a \in A$. This is the probability that a voter would submit preference order in which the correct winner $a$ was in the $k$ th best position.

Definition 20 A social choice rule $F: P(C)^{n} \rightarrow 2^{C}$ is a maximum likelihood estimator (MLE) if there exists a noise model $P$ (not depending on $n$ ) such that

$$
F(R)=\operatorname{argmax}_{x \in C} \prod_{i=1}^{n} P\left(\operatorname{pos}_{v_{i}}(x) \mid x\right)
$$

for any positive integer $n$ and any profile $V=\left(v_{1}, \ldots, v_{n}\right) \in P(C)^{n}$.

Voting rules which fall into this category are MLEWIV (maximum likelihood estimator for winner under identically distributed independent votes) rules in terms of Conitzer and Sandholm (2005).

Let $|C|=m$. A reasonable model of noise in this case is as follows: we introduce a scoring vector $\alpha=\left(\alpha_{1}, \ldots, \alpha_{m}\right)$ whose coordinates are nonnegative, $\alpha_{1} \geq \alpha_{2} \geq \ldots \geq$ $\alpha_{m}$, and set

$$
P(k \mid a)=c e^{-\lambda \alpha_{k}}, \quad c=\left(\sum_{k=1}^{m} e^{-\lambda \alpha_{k}}\right)^{-1} .
$$


Theorem 24 Let $\alpha=\left(\alpha_{1}, \ldots, \alpha_{m}\right)$ be a vector with non-negative coordinates. A $M L E W I V$ rule with a neutral noise model (19) is the scoring rule $\mathcal{R}_{\alpha}$.

Proof Let $v \in P(C)$ and $\operatorname{pos}_{v}(c)=k$. Then as we know the distance from $v$ to the closest vote $v^{\prime}$ that ranks $c$ first is $d\left(v, v^{\prime}\right)=\alpha_{1}-\alpha_{k}$. Then we have

$$
\begin{aligned}
P(V \mid a) & =\prod_{i=1}^{n} P\left(v_{i} \mid a\right)=\prod_{i=1}^{n} c e^{-\lambda \cdot \alpha_{\operatorname{pos}_{v_{i}}(a)}}=c e^{-\lambda \sum_{i=1}^{n} \alpha_{\operatorname{pos}_{v_{i}}(a)}} \\
& =c e^{-\lambda\left(n \alpha_{1}-\sum_{i=1}^{n} d_{\alpha}\left(v_{i}, v_{i}^{\prime}\right)\right)}
\end{aligned}
$$

where $v_{i}^{\prime}$ is the closest (in metric $d_{\alpha}$ ) linear order to $v_{i}$ in which $a$ is the top candidate. Then maximising the right-hand-side of $(20)$ is the same as minimising $\widehat{d_{\alpha}}\left(V, V^{\prime}\right)$ for the weakly unanimous profile $V^{\prime}=\left(v_{1}^{\prime}, v_{2}^{\prime}, \ldots, v_{n}^{\prime}\right)$. Thus our rule is $F\left(\mathcal{U}, \widehat{d_{\alpha}}\right)$ and hence $\mathcal{R}_{\alpha}$ by Theorem 1 .

Therefore we can say that apart from exotic noise models MLERIV voting rules are those that can be $\ell_{1}$-votewise distance rationalised relative to the strong unanimity consensus and MLEWIV voting rules are just scoring rules.

\section{Conclusions and Open Problems}

In this paper we have presented general results regarding the recently introduced distance rationalizability framework. Our paper has three main contributions:

1. We have shown that without any restrictions, essentially every reasonable voting rule is distance-rationalizable and further refinement of this framework is needed.

2. We have put forward a natural class of distances - votewise distances - and proved that the rules which can be distance-rationalized using such distances have several desirable properties. We have identified a number of votewise rules, as well as showed that some rules are not votewise rationalizable with respect to standard consensus classes, and established complexity results for winner determination under votewise rules.

3. We characterized scoring rules as the class of rules distance rationalizable relative to the unanimity consensus and votewise distance over profiles based on a neutral and min-monotone distance over the votes.

Are votewise distances the only natural distances that one should consider? Such distances are based on the assumption that, given an election $E=(C, V)$, if a voter changes her opinion in a minor way, then the resulting election $E^{\prime}=\left(C, V^{\prime}\right)$ must not deviate from $E$ too far. However some rules have discontinuous nature by definition, especially Young's rule which picks the winner of a largest Condorcet-consistent subelection. It is unlikely that such rules can be distance-rationalized via a votewise distance. Indeed, it can be shown that Young's rule and Maximin can be rationalized with respect to $\mathcal{C}$ via fairly intuitive distances that operate on profiles with different numbers of voters: in the case of Maximin we are, essentially, adding voters, and in the case of Young, we are deleting voters. (We omit the definitions of these rules and the construction due to space constraints). However, neither of these rules is known to be votewise rationalizable. Thus, it would be desirable to extend the class of "acceptable" distances to include some non-votewise distances; how to do this is an interesting research direction. 
We mention that our work is closely related to a sequence of papers of Conitzer, Rognlie, Sandholm, and Xia (Conitzer \& Sandholm, 2005; Conitzer et al., 2009) on interpreting voting rules as maximum likelihood estimators. We show that distance rationalizability framework is reacher even if we restrict ourselves with votewise distance rationalizable rules.

\section{References}

Ailon, N., Charikar, M., \& Newman, A. (2008). Aggregating inconsistent information: Ranking and clustering. Journal of the ACM, 55(5), Article 23.

Aleskerov, F., Chistyakov, V., \& Kalyagin, V. (2010). The threshold aggregation. Economic Letters, 107(2), 261-262.

Arrow, K. (1951 (revised editon, 1963)). Social choice and individual values. John Wiley and Sons.

Baigent, N. (1987). Metric rationalisation of social choice functions according to principles of social choice. Mathematical Social Sciences, 13(1), 59-65.

Bauer, F., Stoer, J., \& Witzgall, C. (1961). Absolute and monotonic norms. Numerische Matematic, 3, 257-264.

Bogard, K. (1973). Preference structures i: Distances between transitive preference relations. Journal of Mathematical Sociology, 3, 49-67.

Bogard, K. (1975). Preference structures ii: Distances between transitive preference relations. SIAM Journal of Applied Mathematics, 29, 254-262.

Brams, S., \& Fishburn, P. (2002). Voting procedures. In K. Arrow, A. Sen, \& K. Suzumura (Eds.), Handbook of social choice and welfare, volume 1 (pp. 173-236). Elsevier.

Brandt, F. (2009). Some remarks on Dodgson's voting rule. Mathematical Logic Quarterly, 55(4), 460-463.

Caragiannis, I., Covey, J., Feldman, M., Homan, C., Kaklamanis, C., Karanikolas, N., et al. (2009, January). On the approximability of Dodgson and Young elections. In Proceedings of the 20th annual acm-siam symposium on discrete algorithms (pp. 1058-1067). Society for Industrial and Applied Mathematics.

Caragiannis, I., Kaklamanis, C., Karanikolas, N., \& Procaccia, A. (2010). Socially desirable approximations for Dodgson's voting rule. In Proceedings of the 11th acm conference on electronic commerce. ACM Press. (To appear)

Carpara, A. (1977). Sorting by reversal is difficult. In Proceedins of the first annual international conference on computational molecular biology (recomb'97) (pp. 7583). ACM Press, New York.

Carrizosa, E., \& Fliege, J. (2002). Generalized goal programming: Polynomial methods and applications. Mathematical Programming, 93(2), 281-303.

Chebotarev, P. Y., \& Shamis, E. (1998). Characterizations of scoring methods for preference aggregation. Annals of Operations Research, 80, 299332.

Conitzer, V., Rognlie, M., \& Xia, L. (2009, July). Preference functions that score rankings and maximum likelihood estimation. In Proceedings of the 21st international joint conference on artificial intelligence. AAAI Press.

Conitzer, V., \& Sandholm, T. (2005, July). Common voting rules as maximum likelihood estimators. In Proceedings of the 21st conference in uncertainty in artificial intelligence (pp. 145-152). AUAI Press. 
Conitzer, V., Sandholm, T., \& Lang, J. (2007). When are elections with few candidates hard to manipulate? Journal of the ACM, 54(3), Article 14.

Cook, W., \& Seiford, L. (1978). Priority ranking and consensus information. Management Science, 24, 1721-1732.

Cook, W., \& Seiford, L. (1982). On the Borda-Kendall consensus method for priority ranking problems. Management Science, 28, 621-637.

Coppersmith, D., Fleisher, L., \& Rurda, A. (2010). Ordering by weighted number of wins gives a good ranking for weighted tournaments. , 6(3), Article 55.

Critchlow, D. (1985). Metric methods for analyzing partially ranked data. lecture notes in statistics,34. Springer-Verlag. Berlin.

Deza, M. M., \& Deza, E. (2009). Encyclopedia of distances. Springer.

Diaconis, P. (1988). Group representations in probability and statistics. lecture notesmonograph series, 11. Institut of Mathematical Statistics, Hayward, California.

Dwork, C., Kumar, R., Naor, M., \& Sivakumar, D. (2001, March). Rank aggregation methods for the web. In Proceedings of the 10th international world wide web conference (p. 613-622). ACM Press.

Elkind, E., Faliszewski, P., \& Slinko, A. (2009, July). On distance rationalizability of some voting rules. In Proceedings of the 12th conference on theoretical aspects of rationality and knowledge (pp. 108-117). ACM Press.

Elkind, E., Faliszewski, P., \& Slinko, A. (2011). Rationalizations of condorcetconsistent rules via distances of hamming type. Social Choice and Welfare, DOI: 10.1007/s00355-011-0555-0.

Faliszewski, P., Hemaspaandra, E., \& Hemaspaandra, L. (2009). How hard is bribery in elections? Journal of Artificial Intelligence Research, 35, 485-532.

Faliszewski, P., Hemaspaandra, E., Hemaspaandra, L., \& Rothe, J. (2009). Llull and Copeland voting computationally resist bribery and constructive control. Journal of Artificial Intelligence Research, 35, 275-341.

Hemaspaandra, E., Hemaspaandra, L., \& Rothe, J. (1997). Exact analysis of Dodgson elections: Lewis Carroll's 1876 voting system is complete for parallel access to NP. Journal of the ACM, 44(6), 806-825.

Hemaspaandra, E., Spakowski, H., \& Vogel, J. (2005). The complexity of Kemeny elections. Theoretical Computer Science, 349(3), 382-391.

Hemaspaandra, L., \& Ogihara, M. (2002). The complexity theory companion. SpringerVerlag.

Karp, R., \& Lipton, R. (1980, April). Some connections between nonuniform and uniform complexity classes. In Proceedings of the 12th acm symposium on theory of computing (pp. 302-309). ACM Press. (An extended version has also appeared as: Turing machines that take advice, L'Enseignement Mathématique, 2nd series, 28:191-209, 1982.)

Kemeny, J. (1959). Mathematics without numbers. Daedalus, 88, 577-591.

Kendall, M., \& Gibbons, J. (1990). Rank correlation methods. Oxford University Press.

Kenyon-Mathieu, C., \& Schudy, W. (2007, June). How to rank with few errors. In Proceedings of the 39th acm symposium on theory of computing (pp. 95-103). ACM Press.

Klamler, C. (2005a). Borda and Condorcet: Some distance results. Theory and Decision, 59(2), 97-109.

Klamler, C. (2005b). The Copeland rule and Condorcet's principle. Economic Theory, $25(3), 745-749$. 
Konczak, K., \& Lang, J. (2005, July/August). Voting procedures with incomplete preferences. In Proceedins of the multidisciplinary ijcai-05 worshop on advances in preference handling (pp. 124-129).

Lenstra, Jr., H. (1983). Integer programming with a fixed number of variables. Mathematics of Operations Research, 8(4), 538-548.

Litvak, B. (1982). Information given by the experts. methods of acquisition and analysis. Radio and Communication, Moscow.

Litvak, B. (1983). Distances and consensus rankings. Cybernetics and systems analysis, 19(1), 71-81. (Translated from Kibernetika, No. 1, pp. 57-63, January-February, 1983.)

Mallows, C. (1957). Non-null ranking models. Biometrica, 44, 114-130.

Meir, R., Procaccia, A., Rosenschein, J., \& Zohar, A. (2008). The complexity of strategic behavior in multi-winner elections. Journal of Artificial Intelligence Research, 33, 149-178.

Meskanen, T., \& Nurmi, H. (2008). Closeness counts in social choice. In M. Braham \& F. Steffen (Eds.), Power, freedom, and voting (p. 289-306). Springer-Verlag.

Moulin, H. (1991). Axioms of cooperative decision making. Cambridge University Press.

Nitzan, S. (1981). Some measures of closeness to unanimity and their implications. Theory and Decision, 13(2), 129-138.

Schulze, M. (2003). A new monotonic and clone-independent single-winner election method. Voting Matters, 17, 9-19.

Young, H. (1975). Social choice scoring functions. SIAM Journal on Applied Mathematics, 28(4), 824-838.

Young, H. (1977). Extending Condorcet's rule. Journal of Economic Theory, 16(2), $335-353$.

Young, H., \& Levenglick, A. (1978). A consistent extension of Condorcet's election principle. SIAM Journal on Applied Mathematics, 35(2), 285-300.

\section{Appendix}

\section{A1. $\mathcal{M}$-Borda is not Homogeneous}

Here we present an example showing that Theorem 6 cannot be extended even to additively votewise rules.

Example $2 \mathcal{M}$-Borda is not homogeneous despite being additively votewise. To see this it suffices to consider the following election:

\begin{tabular}{cccccc}
$v_{1}$ & $v_{2}$ & $v_{3}$ & $v_{4}$ & $v_{5}$ & $v_{6}$ \\
\hline$b$ & $a$ & $c$ & $c$ & $c$ & $d$ \\
$a$ & $b$ & $b$ & $b$ & $d$ & $a$ \\
$d$ & $d$ & $a$ & $a$ & $a$ & $b$ \\
$e$ & $e$ & $e$ & $e$ & $e$ & $e$ \\
$c$ & $c$ & $d$ & $d$ & $b$ & $c$
\end{tabular}

A simple calculation shows that to become a majority winner $a$ needs 4 swaps, $b$ needs 3 swaps, $c$ needs 4 swaps, and $d$ needs 5 swaps. Thus, $b$ is a winner according to $\mathcal{M}$-Borda. However, if we replace each voter by two identical ones, it turns out that $b$ needs 5 swaps to become a majority winner, but $c$ requires only 4 and is the $\mathcal{M}$-Borda winner of this new election.

A2. Condorcet Consensus and Homogeneity

The Condorcet consensus is not split-homogeneous as the following example demonstrates. 


\begin{tabular}{cccccc}
$v_{1}$ & $v_{2}$ & $v_{3}$ & $v_{4}$ & $v_{5}$ & $v_{6}$ \\
\hline$c$ & $a$ & $c$ & $b$ & $a$ & $c$ \\
$\downarrow$ & $b$ & $a$ & $\downarrow$ & $\downarrow$ & $\downarrow$ \\
$b$ & $c$ & $b$ & $a$ & $b$ & $a$ \\
$\downarrow$ & $\downarrow$ & $\downarrow$ & $\downarrow$ & $\downarrow$ & $\downarrow$ \\
$a$ & $\downarrow$ & $\downarrow$ & $c$ & $c$ & $b$ \\
$\downarrow$ & $\downarrow$ & $\downarrow$ & $\downarrow$ & $\downarrow$ & $\downarrow$
\end{tabular}

Table 1 Election $E=(C, V)$ from the proof of Proposition 11 .

Example 3 Consider the following election $E=(C, V)$ with $C=\{a, b, c, d, e\}$ and $V=$ $\left(v_{1}, \ldots, v_{12}\right)$

\begin{tabular}{cccccccccccc}
$v_{1}$ & $v_{2}$ & $v_{3}$ & $v_{4}$ & $v_{5}$ & $v_{6}$ & $v_{7}$ & $v_{8}$ & $v_{9}$ & $v_{10}$ & $v_{11}$ & $v_{12}$ \\
\hline$a$ & $b$ & $c$ & $d$ & $e$ & $c$ & $e$ & $a$ & $b$ & $c$ & $d$ & $c$ \\
$b$ & $c$ & $d$ & $e$ & $a$ & $a$ & $d$ & $e$ & $a$ & $b$ & $c$ & $a$ \\
$c$ & $d$ & $e$ & $a$ & $b$ & $b$ & $c$ & $d$ & $e$ & $a$ & $b$ & $b$ \\
$d$ & $e$ & $a$ & $b$ & $c$ & $d$ & $b$ & $c$ & $d$ & $e$ & $a$ & $d$ \\
$e$ & $a$ & $b$ & $c$ & $d$ & $e$ & $a$ & $b$ & $c$ & $d$ & $e$ & $e$
\end{tabular}

Here, voters from the group $V_{1}=\left(v_{1}, \ldots, v_{5}\right)$ form a Condorcet cycle, and voters from the group $V_{2}=\left(v_{7}, \ldots, v_{11}\right)$ are obtained from voters $v_{1}, \ldots, v_{5}$ by reversing their preferences. Voters $v_{6}$ and $v_{12}$ are identical and rank candidate $c$ on top. Since voters from the groups $V_{1}$ and $V_{2}$ 'cancel' each other $c$ is the Condorcet winner in E. On the other hand, consider elections $E_{1}=\left(C, V_{1}\right)$ and $E_{2}=\left(C, V_{2}\right)$. In $E_{1}, b$ is ranked above $c$ in 4 votes, so $c$ is not a Condorcet winner in $E_{1}$. Similarly, in $E_{2}, d$ is ranked above $c$ in 4 votes, so $c$ is not a Condorcet winner in $E_{2}$ either.

There are $\ell_{\infty}$-votewise distances that combined with the Condorcet consensus yield nonhomogeneous rules.

Proposition 11 There exists a set of candidates $C$ and a distance $d$ on $\mathcal{P}(C)$ such that the voting rule rationalized by $\left(\mathcal{C}, \widehat{d^{\infty}}\right)$ is not homogeneous.

Proof We first define two additional types of swap operations for preference orders. A forward distance-two swap of candidate $c$ transforms this preference order as follows: the candidate ranked two positions higher than $c$, is moved from his current position and placed directly below $c$. If $c$ were ranked first or second, a forward distance-two swap is not defined. For example, if $C=\{a, b, c, d, e\}$ and the preference order is $a \succ b \succ c \succ d \succ e$, then the result of a forward distance-two swap of candidate $c$ will be $b \succ c \succ a \succ d \succ e$. A backward distance-two swap is defined similarly. It is easy to see that a single forward distance-two swap can be reversed by applying a single backward distance-two swap and the other way round.

We can now define our distance $d$. Let us fix some candidate set $C=\left\{c_{1}, \ldots, c_{m}\right\}$. For each two preference orders $u$ and $v$ over $C$ we define $d(u, v)$ to be the minimal number of swaps of adjacent candidates and distance-two swaps of candidates needed to transform vote $u$ into vote $v$. It is easy to see that $d$ indeed is a distance because it counts the number of reversible operations that transform one preference order into the other. As before, $\widehat{d^{\infty}}$ is the $\ell_{\infty}$-votewise extension of $d$ to a distance over elections.

Let $\mathcal{R}$ be a voting rule that selects $\left(\mathcal{C}, \widehat{d^{\infty}}\right)$-winners. We will now build an election $E=$ $(C, V)$ such that $\mathcal{R}(E) \neq \mathcal{R}(2 E)$. We set $C=\left\{a, b, c, x_{1}, \ldots, x_{t}\right\}$, where $t$ is a sufficiently large integer. (After reading our description of the votes in $V$ it will become clear what we mean by sufficiently large.) The set of voters $V$ will contain six voters, $v_{1}, \ldots, v_{6}$, whose preference orders are presented in Table 1 . Note that in this table we only showed how candidates in $\{a, b, c\}$ are ranked. Remaining candidates are ranked in the places of arrows, in such a way that (a) each candidate in $\{a, b, c\}$ is preferred to each candidate $x_{i}, 1 \leq i \leq t$, by a majority of voters, and (b) one needs at least three swaps or distance-two swaps to change the relative order of two candidates from $\{a, b, c\}$ that are separated by an arrow.

We have the following results of head-to-head contests in $E$ : four voters prefer $a$ to $b, a$ and $c$ are tied, $b$ and $c$ are also tied. Thus, a single swap of $a$ and $c$ in vote $v_{3}$ makes $a$ a 
Condorcet winner of the election. On the other hand, it is easy to see that being allowed one (possibly distance-two) swap per vote, it is impossible to make either $b$ or $c$ the Condorcet winner. Thus, $a$ is the unique $\mathcal{R}$-winner of $E$.

In $2 E$, similarly, a single swap (within one of the copies of $v_{3}$ ) suffices to make $a$ the Condorcet winner. However, now also a single swap per vote suffices to make $c$ a Condorcet winner. Indeed, in one copy of $v_{2}$ we transform $a \succ b \succ c$ into $a \succ c \succ b$ and in the other into $b \succ c \succ a$. These two transformations allow $c$ to break a tie with both $a$ and $b$, and become the Condorcet winner.

\section{A3. Proof of Theorem 9.}

Let $C=\left\{c_{1}, \ldots, c_{m}\right\}$ be a set of candidates. For two votes $u, v \in P(C)$, we say that $v$ can be obtained from $u$ by a cyclic shift if there exists an $i \in[m]$ and a permutation $\pi: C \rightarrow C$ such that $v=\pi\left(c_{1}\right) \succ \ldots \succ \pi\left(c_{m}\right), u=\pi\left(c_{i}\right) \succ \ldots \succ \pi\left(c_{m}\right) \succ \pi\left(c_{1}\right) \succ \ldots \succ \pi\left(c_{i-1}\right)$.

Partition $P(C)$ into $m$ groups $L_{1}, \ldots, L_{m}$, where the voters in $L_{i}$ rank $c_{i}$ on top. Set $s=(m-1)$ ! and, for each $i \in[m]$, number the votes in $L_{i}$ as $v_{i}^{1}, \ldots, v_{i}^{s}$ so that for any $i, j \in[m]$ the vote $v_{j}^{t}$ can be obtained from the vote $v_{i}^{t}$ by a cyclic shift. This is possible, since for each $u_{i}^{t}, i \in[m], t \in[s]$ and each $j \in[m]$, there is exactly one vote in $L_{j}$ that can be obtained from $v_{i}^{t}$ by a cyclic shift. Now, set $d\left(v_{i}^{t}, v_{j}^{r}\right)=1$ if either $i=j$ or $t=r$, but $(i, t) \neq(j, r)$, and set $d\left(v_{i}^{t}, v_{j}^{r}\right)=2$ if $i \neq j, t \neq r$. Observe that since $d(u, v) \in\{1,2\}$ for $u \neq v$, the mapping $d$ satisfies the triangle inequality; it is also symmetric and neutral.

Consider a preference profile $V$. For any $i \in[m], t \in[s]$, let $a_{i}^{t}$ denote the number of voters in $V$ with preferences $v_{i}^{t}$.

We have

$$
\begin{aligned}
\widehat{d}\left(V, v_{i}^{t}\right) & =\sum_{r \in[s] \backslash\{t\}} a_{i}^{r}+\sum_{j \in[m] \backslash\{i\}} a_{j}^{t}+2 \sum_{j \in[m] \backslash\{i\}} \sum_{r \in[s] \backslash\{t\}} a_{j}^{r} \\
& =\sum_{j \in[m] \backslash\{i\}} \sum_{r \in[s]} a_{j}^{r}+\sum_{j \in[m]} \sum_{r \in[s] \backslash\{t\}} a_{j}^{r} .
\end{aligned}
$$

Consequently, the distance from $V$ to the nearest profile in $\mathcal{S}$ with winner $c_{i}$ is given by $\sum_{j \in[m] \backslash\{i\}} \sum_{r \in[s]} a_{j}^{r}+\min _{t \in[s]} \sum_{j \in[m]} \sum_{r \in[s] \backslash\{t\}} a_{j}^{r}$. The second component of this expression does not depend on $i$, while its first component counts the number of voters that do not rank $c_{i}$ first. Thus, the nearest strong unanimity consensus to $V$ has $c_{i}$ as a winner if and only if $i$ minimizes the sum $\sum_{j \in[m] \backslash\{i\}} \sum_{r \in[s]} a_{j}^{r}$ over all $i \in[m]$, i.e., $c_{i}$ has the largest number of first-place votes. Thus, Plurality is distance-rationalizable with respect to $\mathcal{S}$ via $\widehat{d}$.

Now let us deal with Borda. This construction is for the three candidate set $C=\{a, b, c\}$. Consider a distance $d$ over $P(C)$ satisfying neutrality. By symmetry $d$ is completely described by its values on the pairs $(a b c, a b c),(a b c, a c b),(a b c, b a c),(a b c, b c a),(a b c, c a b)$, and $(a b c, c b a)$. Further, we have $d(a b c, a b c)=0$, and by neutrality and symmetry we have $d(a b c, b c a)=$ $d(a b c, c a b)$ (to see this, note that the permutation $\pi$ given by $\pi(a)=c, \pi(b)=a, \pi(c)=b$ transforms $a b c$ into $c a b$ and $b c a$ into $a b c)$. Set $d(a b c, a c b)=T, d(a b c, b a c)=B, d(a b c, c b a)=M$, $d(a b c, b c a)=d(a b c, c a b)=S$. Table 2 gives the values of $d$ for each pair of preference orders. ${ }^{9}$ Since $d$ is a distance, we have $T, M, B, S>0$.

Not all values of $T, M, B, S$ give us a distance but if all of them are either 1 or 2 , then they will. Suppose that Borda is additively votewise distance rationalizable with respect to $\mathcal{S}$ via a distance $d$ on votes given by some values $T, M, B$, and $S$. Consider two families of preference profiles, $V_{1}(k)$ and $V_{2}(k)$, where $k=1,2, \ldots$ Both $V_{1}(k)$ and $V_{2}(k)$ contain $k$ voters with preference order $a c b, k$ voters with preference order $b c a$, and one extra voter. In the case of $V_{1}(k)$ this extra voter has preference order $c a b$, and in the case of $V_{2}(k)$, the extra voter has

9 If a vote $u$ is obtained from a vote $v$ by permuting the second and the third candidate (and leaving the top candidate in place), we have $d(u, v)=T$, if $u$ is obtained from $v$ by permuting the first and the second candidate (and leaving the bottom candidate in place), we have $d(u, v)=B$, if $u$ is obtained from $v$ by permuting the first and the third candidate (and leaving the center in place), we have $d(u, v)=M$, and if $u$ is obtained from $v$ by a cyclic shift, we have $d(u, v)=S$. 


\begin{tabular}{c|cccccc} 
& $a b c$ & $a c b$ & $b a c$ & $b c a$ & $c a b$ & $c b a$ \\
\hline$a b c$ & 0 & $T$ & $B$ & $S$ & $S$ & $M$ \\
$a c b$ & $T$ & 0 & $S$ & $M$ & $B$ & $S$ \\
$b a c$ & $B$ & $S$ & 0 & $T$ & $M$ & $S$ \\
$b c a$ & $S$ & $M$ & $T$ & 0 & $S$ & $B$ \\
$c a b$ & $S$ & $B$ & $M$ & $S$ & 0 & $T$ \\
$c b a$ & $M$ & $S$ & $S$ & $B$ & $T$ & 0
\end{tabular}

Table 2 The values of $d$ for each pair of votes over $C$.

preference $a c b$. We have

$$
\begin{aligned}
d_{1} & =\widehat{d}\left(V_{1}(k), c a b\right)=k B+k S, \\
d_{2} & =\widehat{d}\left(V_{1}(k), c b a\right)=k S+k B+T, \\
d_{3} & =\widehat{d}\left(V_{1}(k), a c b\right)=k M+B, \\
d_{4} & =\widehat{d}\left(V_{1}(k), b c a\right)=k M+S .
\end{aligned}
$$

Naturally, $d_{1}<d_{2}$. Further, for each $k>0$, the unique winner of $V_{1}(k)$ is $c$, and the unique winner of $V_{2}(k)$ is $a$. Therefore, for any $k>0$ it holds that $d_{1}<d_{3}$, and $d_{1}<d_{4}$, that is, in particular, for each $k$ we have

$$
(k-1) B+k S<k M .
$$

On the other hand, for $V_{2}(k)$ we have:

$$
\begin{aligned}
d_{1}^{\prime} & =\widehat{d}\left(V_{2}(k), c a b\right)=(k+1) B+k S, \\
d_{2}^{\prime} & =\widehat{d}\left(V_{2}(k), c b a\right)=(k+1) S+k B, \\
d_{3}^{\prime}=\widehat{d}\left(V_{2}(k), a c b\right) & =k M, \\
d_{4}^{\prime}=\widehat{d}\left(V_{2}(k), a b c\right) & =(k+1) T+k S .
\end{aligned}
$$

By triangle inequality, $T+S=d(a b c, a c b)+d(a c b, c b a) \geq d(a b c, c b a)=M$, so $d_{3}^{\prime}<d_{4}^{\prime}$. Since $a$ wins in $V_{2}(k)$, we have $d_{3}^{\prime}<d_{1}^{\prime}$ and $d_{3}^{\prime}<d_{2}^{\prime}$. In particular, for each $k>0$ it holds that $k M<(k+1) B+k S$. Combining this inequality with (21), we get that for each $k>0$ it holds that

$$
\frac{k-1}{k} B+S<M<\frac{k+1}{k} B+S .
$$

Since $k$ can be arbitrarily large, we have $M=B+S$.

Now, consider a preference profile $V_{3}=(a b c, a c b)$. Clearly, $a$ is the unique Borda winner for $V_{3}$. We have $d_{1}^{\prime \prime}=\widehat{d}\left(V_{3}, a b c\right)=T, d_{2}^{\prime \prime}=\widehat{d}\left(V_{3}, a c b\right)=T$, and $d_{3}^{\prime \prime}=\widehat{d}\left(V_{3}, c a b\right)=S+B$. Since $a$ is the unique winner, it holds that $T<S+B$. In particular, $T<B$. However, by triangle inequality we know that $T+S \geq M$. We also know that $M=B+S$, so we have $T+S \geq B+S$, that is, $T \geq B$. This is a contradiction. 